TRANSACTIONS OF THE

AMERICAN MATHEMATICAL SOCIETY

Volume 365, Number 4, April 2013, Pages 1901-1931

S 0002-9947(2012)05618-2

Article electronically published on October 11, 2012

\title{
INVARIANTLY UNIVERSAL ANALYTIC QUASI-ORDERS
}

\author{
RICCARDO CAMERLO, ALBERTO MARCONE, AND LUCA MOTTO ROS
}

\begin{abstract}
We introduce the notion of an invariantly universal pair $(S, E)$ where $S$ is an analytic quasi-order and $E \subseteq S$ is an analytic equivalence relation. This means that for any analytic quasi-order $R$ there is a Borel set $B$ invariant under $E$ such that $R$ is Borel bireducible with the restriction of $S$ to $B$. We prove a general result giving a sufficient condition for invariant universality, and we demonstrate several applications of this theorem by showing that the phenomenon of invariant universality is widespread. In fact it occurs for a great number of complete analytic quasi-orders, arising in different areas of mathematics, when they are paired with natural equivalence relations.
\end{abstract}

\section{INTRODUCTION}

Given analytic quasi-orders $R, R^{\prime}$ on standard Borel spaces $X, X^{\prime}$, respectively, say that $R$ Borel reduces to $R^{\prime}$, in symbols $R \leq_{B} R^{\prime}$, if there is a Borel function $f: X \rightarrow X^{\prime}$ such that $\forall x, y \in X\left(x R y \Longleftrightarrow f(x) R^{\prime} f(y)\right)$. Quasi-orders $R, R^{\prime}$ are Borel bireducible if $R \leq_{B} R^{\prime} \leq_{B} R$, in symbols $R \sim_{B} R^{\prime}$. In the sequel we will denote by $E_{R}$ the equivalence relation $R \cap R^{-1}$ induced by a quasi-order $R$.

In LR05, Louveau and Rosendal proved the existence of a complete (sometimes called universal) analytic quasi-order $S$ on a standard Borel space: this means that $R \leq_{B} S$ for every analytic quasi-order $R$ defined on a standard Borel space. Examples of such $S$ can be given in spaces of countable structures (for more details on these spaces, a reference is [Kec95, $\S 16$.C]): if $\mathcal{L}$ is a countable relational language, let $\operatorname{Mod}_{\mathcal{L}}$ be the Polish space of (codes for) $\mathcal{L}$-structures with universe $\omega$, and $j_{\mathcal{L}}$ the logic action of $S_{\infty}$ (the symmetric group of $\omega$ ) on $\operatorname{Mod}_{\mathcal{L}}$. If $\varphi$ is a sentence of $\mathcal{L}_{\omega_{1} \omega}$, then $\operatorname{Mod}_{\varphi}$ will stand for the set of elements of $\operatorname{Mod}_{\mathcal{L}}$ satisfying $\varphi$, and by the Lopez-Escobar theorem (see [Kec95, Theorem 16.8]), the sets of the form $\operatorname{Mod}_{\varphi}$ are exactly the Borel subsets of $\operatorname{Mod}_{\mathcal{L}}$ invariant under $j_{\mathcal{L}}$, i.e. invariant under isomorphism. An example of a complete analytic quasi-order $S$ is then the relation of embeddability for graphs on $\omega$.

In FMR11 Friedman and Motto Ros strengthened this result by showing that for any analytic quasi-order $R$ on a standard Borel space there is a Borel class $B$ of graphs on $\omega$ invariant under isomorphism such that $R$ is Borel bireducible with

Received by the editors March 25, 2010 and, in revised form, May 2, 2011.

2010 Mathematics Subject Classification. Primary 03E15.

Key words and phrases. Analytic equivalence relations, analytic quasi-orders, Borel reducibility, completeness, invariant universality, colored linear orders, dendrites, (ultrametric) Polish spaces, separable Banach spaces.

The first author's research was partially supported by FWF (Austrian Research Fund) through Project number P 19898-N18. The third author's research was supported by FWF through Project number P 19898-N18. The second author's research was partially supported by FWF through Project number P 19898-N18 and by PRIN of Italy.

(C)2012 American Mathematical Society Reverts to public domain 28 years from publication 
the relation of embeddability restricted to $B$. This situation suggests the following definition.

Definition 1.1. Let $S$ be an analytic quasi-order on some standard Borel space $X$, and let $E$ be an analytic equivalence subrelation of $S$ (so that $E \subseteq E_{S}$ ). We say that the pair $(S, E)$ is invariantly universal for analytic quasi-orders if for any analytic quasi-order $R$ there is a Borel subset $B \subseteq X$ invariant with respect to $E$ such that the restriction of $S$ to $B$ is Borel bireducible with $R$.

Similarly, if $F, E$ are analytic equivalence relations on $X$ such that $E \subseteq F$, the pair $(F, E)$ is invariantly universal for analytic equivalence relations if for every analytic equivalence relation $R$ there is a Borel $E$-invariant subset $B \subseteq X$ such that the restriction of $F$ to $B$ is Borel bireducible with $R$.

Invariant universality is a natural strengthening of completeness: in fact, if $(S, E)$ is a pair which is invariantly universal for the class of analytic quasi-orders, then $S$ must be complete for the same class (and similarly for pairs of the form $(F, E)$ ). Despite the fact that invariant universality looks as a very strong notion, the results of this paper show that it is a widespread phenomenon. Moreover, it is somehow related to the notions of completeness with respect to some natural strengthenings of Borel reducibilty, called faithful Borel reducibility and classwise Borel embeddability (introduced in [FS89] and MR11, respectively). For example, if $F$ is complete with respect to classwise Borel embeddability for the class of all analytic equivalence relations, then the pair $(F, F)$ is invariantly universal for the same class.

It should also be noted here that invariant universality is an example of the descriptive set theoretical phenomenon of universality, as opposed to the notion of completeness. Generally speaking, if $\Gamma$ is a class of mathematical objects and $X \in \Gamma$, we say that $X$ is $\Gamma$-complete if each element of $\Gamma$ is in some specific sense reducible to $X$, while we say that $X$ is $\Gamma$-universal if it contains in a "natural" way a copy of every element of $\Gamma$. Now let $\Gamma$ be the class of all analytic quasi-orders (or, respectively, of all analytic equivalence relations). If we use $\leq_{B}$ as the reducibility notion, then $\Gamma$-completeness coincides with the notion of completeness introduced at the beginning of this section. On the other hand, in most practical cases one could arguably view the property of "being Borel bireducible with the restriction of $S$ to a Borel $E$-invariant set" as a translation in our context of the property of "having a copy naturally contained in $S$ ": with this identification, $\Gamma$-universality coincides with invariant universality. This will be further clarified by the concrete examples given in Section 5 .

Notice that if $(S, E)$ is invariantly universal for quasi-orders, then $\left(E_{S}, E\right)$ is invariantly universal for equivalence relations. So we shall confine our attention to the former notion, as all results will have a counterpart in the equivalence relation setting.

An elementary property to be noted here is that if the pair $(S, E)$ is universal and $F$ is an equivalence subrelation of $E$, then $(S, F)$ too is universal.

To give a first example of an invariantly universal pair, fix $W \subseteq{ }^{\omega} 2 \times{ }^{\omega} 2 \times{ }^{\omega} 2$ an analytic set whose sections are exactly all analytic quasi-orders on ${ }^{\omega} 2$. Now define

$$
(x, y) S\left(x^{\prime}, y^{\prime}\right) \Longleftrightarrow x=x^{\prime} \wedge\left(x, y, y^{\prime}\right) \in W ;
$$

this is the first example of a complete analytic quasi-order presented in [LR05]. Now, the pair $\left(S, E_{S}\right)$ is easily seen to be invariantly universal. Hence, for every 
analytic equivalence relation $E \subseteq E_{S}$, the pair $(S, E)$ is invariantly universal as well.

In this paper we show that many complete analytic quasi-orders, paired with natural equivalence relations, are invariantly universal. To see however that this is not always the case, consider an analytic non-Borel subset $A$ of a standard Borel space $X$ and a complete analytic quasi-order $S$ on $X$. Define the analytic quasiorder $R$ on $X \times X$ by

$$
(x, y) R\left(x^{\prime}, y^{\prime}\right) \Longleftrightarrow(x, y)=\left(x^{\prime}, y^{\prime}\right) \vee\left(x, x^{\prime} \in A \wedge y S y^{\prime}\right)
$$

and the analytic equivalence relation $E$ by

$$
(x, y) E\left(x^{\prime}, y^{\prime}\right) \Longleftrightarrow y=y^{\prime} \wedge\left(x, x^{\prime} \in A \vee x=x^{\prime}\right)
$$

Then $R$ is a complete analytic quasi-order, but $(R, E)$ (so neither $\left(R, E_{R}\right)$ ) is not invariantly universal, as the restriction of $R$ to any Borel $E$-invariant set is either non-Borel or a smooth equivalence relation.

The paper consists of two parts. The first one culminates with the proof of Theorem 4.2, the main result of the paper, which gives a widely applicable sufficient condition for a pair $(S, E)$ to be invariantly universal. This condition might appear somewhat technical; however, it turns out to be quite powerful. In fact, it will be our tool for giving many examples of invariantly universal pairs in the second part of the article. These include quasi-orders from model theory, combinatorics, topology, metric space theory, and separable Banach space theory. Notice that each of these results of invariant universality can also be interpreted as a characterization of the class of analytic quasi-orders and equivalence relations, as if $(S, E)$ is invariantly universal (where $S, E$ are defined on a standard Borel space $X$ ), then a binary relation $R$ defined on a standard Borel space is an analytic quasi-order (resp. an analytic equivalence relation) if and only if there is a Borel $E$-invariant $B \subseteq X$ such that $R \sim_{B} S \uparrow B$ (resp. $R \sim_{B} E_{S} \uparrow B$ ).

We warmly thank Christian Rosendal, to whom we are indebted for several illuminating discussions. These eventually led to our results on separable Banach spaces contained in subsection 5.6 .

\section{SOME PRELIMINARIES AND NOTATION}

A typical example of a pair $(S, E)$ which will be considered in this paper is the following: suppose that $A \subseteq{ }^{\omega} \omega \times X \times X$ (where ${ }^{\omega} \omega$ is the Baire space consisting of all functions from $\omega$ into itself) is Borel and such that for all $x, y, z \in X$

- $(i d, x, x) \in A$,

- $(f, x, y) \in A \wedge(g, y, z) \in A \Rightarrow(g \circ f, x, z) \in A$.

Then the relation $S=\left\{(x, y) \in X^{2} \mid \exists f: \omega \rightarrow \omega(f, x, y) \in A\right\}$ is an analytic quasi-order, which we will call a morphism relation. The equivalence relation

$$
E=\left\{(x, y) \in X^{2} \mid \exists f \in S_{\infty}\left((f, x, y) \in A \wedge\left(f^{-1}, y, x\right) \in A\right)\right\}
$$

will be called the $A$-isomorphism relation or, with some abuse of terminology, the $S$ isomorphism relation. This is usually a minor abuse, as in all cases we shall consider there is a natural $A$ which generates $S$. The equivalence relation $E_{S}$ induced by $S$, which clearly satisfies $E \subseteq E_{S}$, will be called bimorphism relation.

A natural context in which such morphism relations appear is when considering the quasi-order $S$ induced by some model theoretic notions of morphism (like e.g. embedding, homomorphism, weak-homomorphism, or epimorphism) on some 
$\operatorname{Mod}_{\psi}$ : in all these cases the $S$-isomorphism relation associated with $S$ is simply the isomorphism relation $\cong$ so that, again by the Lopez-Escobar theorem, such an $S$ is invariantly universal if and only if for every analytic quasi-order $R$ there is an $\mathcal{L}_{\omega_{1} \omega}$-sentence $\varphi$ such that $R$ is Borel bireducible with $S$ restricted to $\operatorname{Mod}_{\varphi}$.

The terminology given above will be extended in a natural way to many other kinds of relations we shall consider, and when dealing with a pair $(S, E)$ given by a morphism relation and its associated $S$-isomorphism relation, we will briefly say that the quasi-order $S$ is invariantly universal. Using this terminology, we can reformulate some results of [FMR11] (one of which was mentioned in the introduction) by saying:

Theorem 2.1. The relations of embeddability and of homomorphism for graphs on $\omega$ are both invariantly universal.

We now fix some notation and recall some results for later use. Given a Polish space $X$, the standard Borel space of closed subsets of $X$ will be denoted by $F(X)$. If $X$ is a Polish group, $G(X)$ will stand for the standard Borel space of closed subgroups of $X$. The orbit equivalence relation induced by an action $a$ will be denoted $E_{a}$.

For $t$ a finite sequence, $|t|$ will denote its length. Given an element $u$ and a natural number $n, u^{n}$ will indicate the sequence of length $n$ and constant value $u$. Starting with a linearly ordered set, we shall denote by $\leq_{\text {lex }}$ the lexicographic order on the set of finite sequences. If two sequences $s, t \in{ }^{<\omega} \omega$ have the same length, we put $s \leq t$ if $s(i) \leq t(i)$ for every $i<|s|=|t|$, and define $s+t \in{ }^{<\omega} \omega$ by setting $(s+t)(i)=s(i)+t(i)$. Given a non-empty set $X$ and a tree $T$ on $X \times \omega$, we say that $T$ is normal if given $u \in{ }^{<\omega} X$ and $s \in{ }^{<\omega} \omega,(u, s) \in T$ implies $(u, t) \in T$ for every $s \leq t$.

It is well known that any analytic subset of ${ }^{\omega} 2 \times{ }^{\omega} 2$, in particular any analytic quasi-order $R$ on ${ }^{\omega} 2$, is the projection $p$ of the body of a tree on $2 \times 2 \times \omega$. In LR05, Theorem 2.4], this property is strengthened by showing that such an $R$ can be viewed as the projection of the body of a normal tree $S$ on $2 \times 2 \times \omega$ such that the reflexivity and transitivity properties of $R$ are mirrored by corresponding local properties of $S$. A further strengthening, useful in applications, was isolated in FMR11. Wrapping up, we have the following.

Proposition 2.2 (LR05, FMR11]). Let $R$ be any analytic quasi-order on ${ }^{\omega} 2$. Then there is a normal tree $S$ on $2 \times 2 \times \omega$ such that:

i) $R=p[S]$;

ii) for every $u \in<^{<\omega_{2}}$ and $s \in{ }^{<\omega} \omega$ of the same length, $(u, u, s) \in S$;

iii) for every $u, v, w \in{ }^{<\omega} 2$ and $s, t \in{ }^{<\omega} \omega$ of the same length, if $(u, v, s) \in S$ and $(v, w, t) \in S$, then $(u, w, s+t) \in S$

iv) for every $u, v \in{ }^{<\omega} 2$ of the same length, $\left(u, v, 0^{|u|}\right) \in S$ implies $u=v$.

A function $f:{ }^{<\omega} \omega \rightarrow{ }^{<\omega} \omega$ is said to be Lipschitz if $s \subseteq t \Rightarrow f(s) \subseteq f(t)$ and $|s|=|f(s)|$ for each $s, t \in{ }^{<\omega} \omega$. Now consider the space $\mathcal{T}$ of all normal trees on $2 \times \omega$, and for $S, T \in \mathcal{T}$ put $S \leq_{\max } T$ if and only if there exists a Lipschitz function $f:{ }^{<\omega} \omega \rightarrow{ }^{<\omega} \omega$ such that $(u, s) \in S \Rightarrow(u, f(s)) \in T$ for every $u \in{ }^{<\omega} 2$ and $s \in{ }^{<\omega} \omega$ of the same length (this in particular implies $p[S] \subseteq p[T]$ ).

Theorem 2.3 ([LR05]). The quasi-order $\leq_{\max }$ is complete for analytic quasiorders. 
Indeed, consider an arbitrary analytic quasi-order $R$ on ${ }^{\omega} 2$ together with any tree $S$ satisfying Proposition 2.2 then define for every $x \in{ }^{\omega} 2$ the normal tree $S^{x}=\{(u, s) \mid(u, x \uparrow|u|, s) \in S\}$. It follows that $x R y$ if and only if $S^{x} \leq_{\max } S^{y}$ (for $x, y \in \omega^{\omega} 2$ ). Moreover, as discussed in [FMR11, the map which sends $x$ to $S^{x}$ is continuous and injective. Given a quasi-order $R$ on ${ }^{\omega} 2$ and $x \in{ }^{\omega} 2$, from now on we will denote by $S^{x}$ the normal tree defined as above.

\section{Construction of Sufficiently Rigid trees}

This section contains an adaptation of a construction from [FMR11, which will be needed in the sequel. The possibility of such a construction was already noted in [FMR11, but we will explicitly give definitions and related proofs here for the sake of completeness. The symbol $\sqsubseteq$ will denote embeddability between countable combinatorial trees (that is, connected acyclic graphs).

Let $\#:<\omega \omega \rightarrow \omega$ be any bijection, and let $\theta:<\omega 2 \rightarrow \omega$ be a bijection such that $|u|<|v|$ implies $\theta(u)<\theta(v)$.

Let $G_{0}$ be the combinatorial tree obtained from $<\omega \omega$ by splitting each edge linking $s \uparrow(|s|-1)$ to $s$ by inserting a new vertex $s^{*}$ in between, for $s \in<\omega \omega \backslash$ $\{\emptyset\}$. Next, define a combinatorial tree $G_{1}$ by adding to $G_{0}$ new vertices $s^{+}, s^{++}$, $\left(s^{++}, i, j\right)$, for $s \in{ }^{<\omega} \omega, 0 \leq j \leq i \leq \# s+2$, and then link $s$ to $s^{+}, s^{+}$to $s^{++}, s^{++}$ to $\left(s^{++}, i, 0\right)$, and $\left(s^{++}, i, j\right)$ to $\left(s^{++}, i, j+1\right)$ whenever these vertices are defined (see Figure 1).

Given a normal tree $T$ on $2 \times \omega$, we define a combinatorial tree $G_{T}$ starting from $G_{1}$ as follows: for every $u \in<\omega_{2}$ and $s \in<\omega \omega$ such that $(u, s) \in T$, add to $G_{1}$

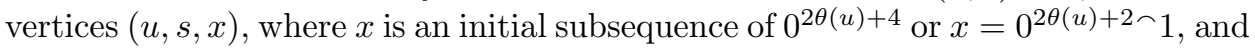
connect $(u, s, \emptyset)$ to $s$ and $(u, s, x)$ to $\left(u, s, x^{\prime}\right)$ just in case one of $x, x^{\prime}$ is an immediate successor of the other. Now it is easy to see how to reprove [LR05, Theorem 3.1], i.e. that $S \leq_{\max } T \Longleftrightarrow G_{S} \sqsubseteq G_{T}$. For one direction, assume first that $S, T$ are normal trees on $2 \times \omega$ such that $S \leq_{\max } T$ : we claim that this can be witnessed by an injective Lipschitz function $f:{ }^{<} \omega \omega \rightarrow{ }^{<} \omega \omega$ such that $\forall s \in<{ }^{<} \omega(\# s \leq \# f(s))$. To see this, let $f^{\prime}:<\omega \omega \rightarrow<\omega \omega$ be any Lipschitz function witnessing $S \leq_{\max } T$, and define inductively $f(s)$ to be the $\leq_{\text {lex }} t$ such that:

- $t$ extends $f(s \uparrow(|s|-1))$ if $|s|>0$,

- $f^{\prime}(s) \leq t$

- $f\left(s^{\prime}\right) \neq t$ for every $s^{\prime} \in|s| \omega$ such that $s^{\prime}<_{\operatorname{lex}} s$,

- $\# s \leq \# t$.

If $f$ is as above, then define the embedding $g$ from $G_{S}$ to $G_{T}$ by sending $s$ to $f(s)$, $s^{*}$ to $f(s)^{*}, s^{+}$to $f(s)^{+}, s^{++}$to $f(s)^{++},\left(s^{++}, i, j\right)$ to $\left(f(s)^{++}, i, j\right)$, and $(u, s, x)$ to $(u, f(s), x)$. For the other direction, assume $S \neq \emptyset$. Notice that all the points in $G_{S}$ have valence $\leq 2$ except for those of the form $s \in<\omega \omega$ (which have valence $\omega$ ), $s^{++}$ (which have valence $\# s+4$ ), and $\left(u, s, 0^{2 \theta(u)+2}\right.$ ) (which have valence 3 ). Moreover, the distance from $s$ to $s^{++}$is always 2 and vertices of the form $\left(u, s, 0^{2 \theta(u)+2}\right)$ have odd distance (greater than 2 ) from vertices in $<\omega \omega$, this distance being determined by function $\theta$, which is increasing with respect to the length of the argument. So if $g$ is an embedding of $G_{S}$ in $G_{T}$, it follows that $g\left(\emptyset, \emptyset, 0^{2 \theta(\emptyset)+2}\right)=\left(\emptyset, \emptyset, 0^{2 \theta(\emptyset)+2}\right)$, and hence $g(\emptyset)=\emptyset$. Now, by induction, conclude that $f=g \uparrow<\omega \omega$ is such that $f(\emptyset)=\emptyset$, range $(f) \subseteq{ }^{<\omega} \omega$, and $f$ witnesses $S \leq_{\max } T$, similarly as in the proof of LR05, Theorem 3.1]. 


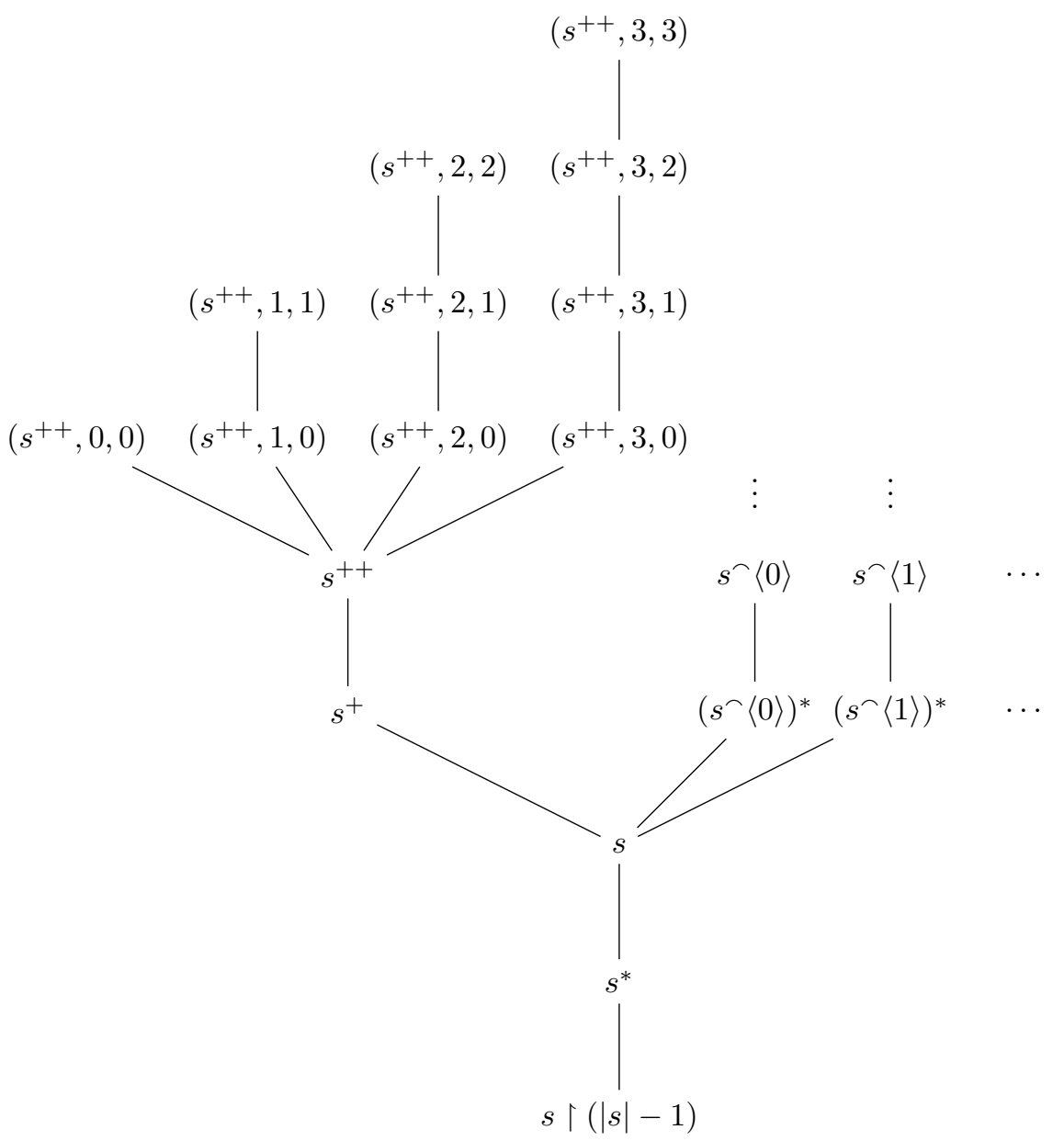

Figure 1. A portion of $G_{1}$ around the unique $s$ such that $\# s=1$.

Lemma 3.1. Let $S, T$ be normal trees on $2 \times \omega$, and $G_{S}$ and $G_{T}$ be defined as before. If $S \neq T$, then $G_{S} \neq G_{T}$.

Proof. Assume that $i$ is an isomorphism between $G_{S}$ and $G_{T}$ in order to show $S \subseteq T$ (and symmetrically $T \subseteq S$ ). Since $i$ preserves distances and valences, each $s^{++}$must be mapped to itself, as it is the unique point in both graphs with valence $\# s+4$. Therefore also $s$ is mapped to itself, as it is the unique point (in both graphs) which is at distance 2 from $s^{++}$and has valence $\omega$. Therefore $i \uparrow<\omega \omega$ is the identity. Now suppose $(u, s) \in S$ : as in the proof of [LR05, Theorem 3.1], the point $\left(u, s, 0^{2 \theta(u)+2}\right)$ must be sent by $i$ to $\left(u, i(s), 0^{2 \theta(u)+2}\right)=\left(u, s, 0^{2 \theta(u)+2}\right)$, which means that $(u, s) \in T$. Hence $S \subseteq T$.

From now on $\mathcal{L}$ will be the language with a single binary relation symbol. Then each $G_{T}$ defined as above will be viewed as an element of $\operatorname{Mod}_{\mathcal{L}}$ (it is easy to see that each $G_{T}$ can be coded Borel-in- $T$ as a graph on $\omega$ ). We shall denote by $\mathbb{G}$ the 
set of all such $G_{T}$. The following corollary gathers two important consequences of (the proof of) Lemma 3.1

Corollary 3.2. $\quad 1) \mathbb{G}$ is a Borel subset of $\operatorname{Mod}_{\mathcal{L}}$, so it is a standard Borel space.

2) On $\mathbb{G}$ equality and isomorphism coincide.

The restrictions to $\mathbb{G}$ of binary relations defined on combinatorial trees (like equality or embeddability) will often be denoted with a subscript $\mathbb{G}$.

Lemma 3.3. For every distinct $p, q \in S_{\infty}$ and every normal tree $T$ on $2 \times \omega$, we have $j_{\mathcal{L}}\left(p, G_{T}\right) \neq j_{\mathcal{L}}\left(q, G_{T}\right)$.

Proof. Assume that $j_{\mathcal{L}}\left(p, G_{T}\right)=j_{\mathcal{L}}\left(q, G_{T}\right)$ : we want to show that in this case $p=q$. To see this first check that if $\left(g_{0}, \ldots, g_{n}\right)$ is a path in $G_{T}$, and $p\left(g_{0}\right)=q\left(g_{0}\right)$ and $p\left(g_{n}\right)=q\left(g_{n}\right)$, then $p\left(g_{k}\right)=q\left(g_{k}\right)$ for every $k \leq n$ (using the acyclicity of $\left.G_{T}\right)$. Therefore, in our case it will be enough to show that $p$ and $q$ coincide on

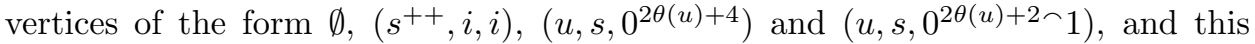
amounts to showing that each of these points is the unique element of $G_{T}$ which satisfies a certain property that can be expressed in terms of valence and distance. First note that $p$ and $q$ must coincide on vertices of the form $s^{++}$, as these are the unique points with valence $\# s+4$ : therefore we get that $p$ and $q$ must also coincide on elements of the form $s \in{ }^{<\omega} \omega$ as $s$ is the unique point of $G_{T}$ which has valence $\omega$ and is at distance 2 from $s^{++}$(in particular we have $p(\emptyset)=q(\emptyset)$ ). Then $p$ and $q$ must coincide on elements of the form $\left(s^{++}, i, i\right)$, as these are the unique points of $G_{T}$ with valence 1 , distance $i+1$ from $s^{++}$, and distance $i+3$ from $s$, and on elements of the form $\left(u, s, 0^{2 \theta(u)+2}\right)$ because these are the unique points with valence 3 , distance $2 \theta(u)+3$ from $s$, and greater distance from any $s^{\prime} \in{ }^{<\omega} \omega$ distinct from $s$. Finally, $p$ and $q$ must coincide on elements of the form $\left(u, s, 0^{2 \theta(u)+4}\right)$ or $\left(u, s, 0^{2 \theta(u)+2 \frown} 1\right)$, as these are the unique points with valence 1 which are at distance 2 and 1 , respectively, from $\left(u, s, 0^{2 \theta(u)+2}\right)$.

Corollary 3.4. Let $T$ be a normal tree on $2 \times \omega$. Then $G_{T}$ is rigid (i.e. its unique automorphism is the identity).

\section{THE MAIN THEOREM}

Theorem 4.2 below constitutes our main tool for proving invariant universality of analytic quasi-orders. To establish it, we have to deal with the problem of $E$ saturating in a Borel way the range of a reduction between embeddability on $\mathbb{G}$ and an analytic quasi-order $S$ with $E$ an equivalence subrelation of $E_{S}$.

First we need the following technical lemma, which is essentially a reformulation of BK96, Theorem 1.2.4]. Let $Y$ be a Polish group and $Z$ a closed subgroup of $Y$. By a theorem of Burgess (see [Kec95, Theorem 12.17]), there is a Borel selector $s: Y \rightarrow Y$ for the equivalence relation on $Y$ whose classes are the (left) cosets of $Z$, and, consequently, a Borel transversal $T=\{y \in Y \mid s(y)=y\}=\operatorname{range}(s)$ for the same equivalence relation. The next lemma is a parametrized version of Burgess' theorem.

Lemma 4.1. Let $X$ be a standard Borel space, $Y$ a Polish group, and $\Sigma: X \rightarrow$ $G(Y)$ a Borel map. Then there is a Borel function $s: X \times Y \rightarrow Y$ such that $s_{x}: Y \rightarrow Y: y \mapsto s(x, y)$ is a selector for the equivalence relation $E_{x}$ whose classes 
are the (left) cosets of $\Sigma(x)$. Therefore $T=\left\{(x, y) \in X \times Y \mid s_{x}(y)=y\right\}$ is Borel as well, and $T_{x}=\{y \in Y \mid(x, y) \in T\}$ is a Borel transversal for $E_{x}$.

Proof. Define $s=d \circ f_{1} \circ f_{0}$ where:

- $f_{0}: X \times Y \rightarrow G(Y) \times Y$ is defined by $f_{0}(x, y)=(\Sigma(x), y)$;

- $f_{1}: G(Y) \times Y \rightarrow F(Y)$ is defined by $f_{1}(G, y)=y G$;

- $d: F(Y) \rightarrow Y$ is a Borel function such that $\forall F \in F(Y) \backslash\{\emptyset\}(d(F) \in F)$.

It is clear that each $s_{x}$ is a selector for $E_{x}$. To see that $s$ is Borel simply notice that $f_{0}$ is Borel because $\Sigma$ is such, while $f_{1}$ is Borel because if $\left\langle d_{n} \mid n \in \omega\right\rangle$ is any sequence of Borel functions from $F(Y)$ into $Y$ such that $\left\langle d_{n}(F) \mid n \in \omega\right\rangle$ is a dense subset of every $F \in F(Y) \backslash\{\emptyset\}$, then

$$
y G=F \Longleftrightarrow \forall n \in \omega\left(y d_{n}(G) \in F \wedge y^{-1} d_{n}(F) \in G\right) .
$$

Theorem 4.2. Let $S$ be an analytic quasi-order on a standard Borel space $Z$, and let $E \subseteq E_{S}$ be an analytic equivalence relation on the same space. Suppose there exists a Borel function $f: \mathbb{G} \rightarrow Z$ which simultaneously witnesses $\sqsubseteq_{\mathbb{G}} \leq_{B} S$ and $={ }_{\mathbb{G}} \leq_{B} E$ (which is the same as $\cong_{\mathbb{G}} \leq_{B} E$ ). Furthermore, let $Y$ be a Polish group, let $a$ be a Borel action of $Y$ on a standard Borel space $W$, and let $g: Z \rightarrow W$ witness $E \leq_{B} E_{a}$.

Consider the map $\Sigma: \mathbb{G} \rightarrow G(Y)$ which assigns to $G \in \mathbb{G}$ the stabilizer of $(g \circ f)(G)$ with respect to a, i.e.

$$
\Sigma(G)=\{y \in Y \mid a(y,(g \circ f)(G))=(g \circ f)(G)\} .
$$

If $\Sigma$ is Borel, then the pair $(S, E)$ is invariantly universal.

Proof. We start by showing that it is enough to prove the following claim.

Claim 4.2.1. For any Borel $B \subseteq \mathbb{G}$, the $E$-saturation $\operatorname{Sat}(f(B))$ of $f(B)$ is Borel.

Granting this claim, the proof of the theorem can be completed as follows: Let $R$ be an arbitrary analytic quasi-order on ${ }^{\omega} 2$, and let $S^{x}$ be normal trees defined as before, so that $x R y \Longleftrightarrow S^{x} \leq_{\max } S^{y} \Longleftrightarrow G_{S^{x}} \sqsubseteq_{\mathbb{G}} G_{S^{y}}$ and the map $x \mapsto G_{S^{x}}$ is injective. Now consider $B_{R}=\left\{G_{S^{x}} \mid x \in{ }^{\omega} 2\right\}$ : being a Borel subset of $\mathbb{G}$, from the claim we get that $C=\operatorname{Sat}\left(f\left(B_{R}\right)\right)$ is Borel and $E$-invariant. The map $\omega_{2} \rightarrow Z: x \mapsto f\left(G_{S^{x}}\right)$ is clearly a reduction of $R$ to $S\lceil C$. For the other direction, note that $f\left(B_{R}\right)$ consists of $E$-incomparable elements (this is by our assumption that $f$ reduces equality on $\mathbb{G}$ to $E$ ), so that the map which sends $y \in C$ to the unique $x \in{ }^{\omega} 2$ such that $f\left(G_{S^{x}}\right) E y$ is a well-defined reduction of $S\lceil C$ to $R$, and it is Borel as its graph is analytic.

It remains to prove the claim. Since $\operatorname{Sat}(f(B))$ is easily seen to be analytic, it is enough to show that it is also a co-analytic set. Apply Lemma 4.1 to the map $\Sigma \uparrow B$, and let $T$ be the resulting Borel subset of $B \times Y$. Since

$$
P=\{z \in Z \mid \exists !(G, y) \in B \times Y((G, y) \in T \wedge a(y, g(f(G)))=g(z))\}
$$

is the set of uniqueness of a Borel set, and is hence co-analytic by a classical result of Luzin (see e.g. [Kec95, Theorem 18.11]), it is enough to show that $\operatorname{Sat}(f(B))=P$. One inclusion is obvious, as if $g(z)=a(y, g(f(G)))$ for some $G \in B$ and $y \in Y$, then $z E f(G)$ and hence $z \in \operatorname{Sat}(f(B))$. For the other direction, assume that $G \in B$ is such that $z E f(G)$. Since $g$ reduces $E$ to $E_{a}$, there is $\bar{y} \in Y$ such that $a(\bar{y}, g(f(G)))=g(z)$. Let $y \in Y$ be in the same (left) coset of $\Sigma(G)$ of $\bar{y}$ and such that $(G, y) \in T$ (such a $y$ must exist because the vertical section $T_{G}$ meets all left 
cosets of $\Sigma(G))$ : since $\bar{y}^{-1} y \in \Sigma(G)$ we have that $a\left(\bar{y}^{-1} y, g(f(G))\right)=g(f(G))$, whence $a(y, g(f(G)))=a(\bar{y}, g(f(G)))=g(z)$. So $(G, y) \in B \times Y$ is such that $(G, y) \in T$ and $a(y, g(f(G)))=g(z)$ : we want to prove that $(G, y)$ is also the unique pair satisfying these conditions, so that $z \in P$. Assume that $\left(G^{\prime}, y^{\prime}\right) \in T$ is such that $a\left(y^{\prime}, g\left(f\left(G^{\prime}\right)\right)\right)=g(z)$. The last condition implies $f\left(G^{\prime}\right) E z E f(G)$, so that $G=G^{\prime}$. But then $a\left(y^{\prime}, g(f(G))\right)=g(z)=a(y, g(f(G)))$ implies that $y$ and $y^{\prime}$ are in the same (left) coset of $\Sigma(G)$ : since $(G, y),\left(G, y^{\prime}\right) \in T$ and $T_{G}$ is a transversal for the equivalence relation on $Y$ whose classes are the (left) cosets of $\Sigma(G)$, we also get that $y=y^{\prime}$, so that $\left(G^{\prime}, y^{\prime}\right)=(G, y)$.

Remark 4.3. Notice that our proof of Theorem 4.2 actually shows that, given $S, Z$ and $f$ as in the hypotheses of such theorem, Claim 4.2 .1 already implies that the pair $(S, E)$ is invariantly universal, and hence such a condition could also be useful in dealing with the case when $E$ is not Borel reducible to an orbit equivalence relation (see e.g. the observation after Question 6.4 below).

Moreover, as kindly pointed out by the anonymous referee, given $S, Z, f, E_{a}$, and $g$ as in the hypotheses of Theorem $4.2, \Sigma$ is a Borel function if and only if the $E_{a^{-}}$ saturation of $g(f(\mathbb{G}))$ is Borel 1$]$ As demonstrated by the applications in Section 5 . despite the fact that the last requirement could seem easier to check, the only way we found to achieve this goal was exactly the content of Theorem 4.2, i.e. to prove that the map $\Sigma$ is Borel.

To apply Theorem 4.2 we thus have to perform three steps:

(a) Find $f: \mathbb{G} \rightarrow Z$ and check that it witnesses both $\sqsubseteq_{\mathbb{G}} \leq_{B} S$ and $=_{\mathbb{G}} \leq_{B} E$. In all applications, $S$ is already known to be a complete analytic quasi-order by a proof showing $\sqsubseteq_{\mathbb{G}} \leq_{B} S$. Thus $f$ will be the function defined in that proof (or a minor modification of it), and this step will consist in checking that such $f$ witnesses also $=_{\mathbb{G}} \leq_{B} E$, usually performing a detailed analysis of $f$.

(b) Find the appropriate $Y, W, a$, and $g$. Often $E$ itself is an orbit equivalence relation of a Polish group action, so that $W=Z, Y$ and $a$ are given, and we tacitly assume that $g$ is the identity.

(c) Prove that $\Sigma$ is Borel. In most applications this is the most delicate part of the proof.

\section{Applications}

In this section we apply Theorem 4.2 to show that all analytic quasi-orders we know to be complete are in fact invariantly universal when coupled with natural equivalence relations. In particular, we answer affirmatively some questions posed in [FMR11, Questions 6 and 8] (although in the first case we have just a partial answer, as the case of epimorphisms preserving relations and functions in both directions still remains completely open). These asked whether the quasi-orders induced by epimorphisms between countable structures, isometric embeddings between ultrametric Polish spaces, continuous embeddings between compact metrizable spaces

\footnotetext{
${ }^{1}$ One direction of the equivalence can be obtained using an argument similar to the proof of Claim 4.2.1 given above. For the other direction, since $g \circ f$ is injective on $\mathbb{G}$, we get that $g(f(\mathbb{G}))$ is a Borel transversal for the restriction of $E_{a}$ to its $E_{a}$-saturation (which is a Borel set by hypothesis). This implies that such a restriction is a smooth orbit equivalence relation and hence, in particular, it is Borel. Let $\sigma$ be the map assigning to each $w$ in the $E_{a}$-saturation of $g(f(\mathbb{G}))$ its stabilizer. Then $\sigma$ is Borel, and hence $\Sigma=\sigma \circ g \circ f$ is also a Borel map.
} 
and linear isometric embeddings between separable Banach spaces are invariantly universal when paired with, respectively, isomorphism, isometry, homeomorphism, and linear isometry.

The section is quite long and contains a great variety of applications in many areas of mathematics, most of them involving some specific new idea or technique. Subsection 5.1 deals with the relation of being epimorphic image between graphs, and constitutes the first non-trivial application of Theorem 4.2. Subsection [5.2 gives some natural examples of invariantly universal quasi-orders in the realm of combinatorics. Subsection 5.3 uses the results from the preceding subsection to give other combinatorial examples which are in turn used in subsection 5.4, where we study invariant universality in topology (this is also the first application of Theorem 4.2 where the analytic equivalence relation under consideration is not an orbit equivalence relation itself, but is only reducible to such a relation). Subsection 5.5 provides applications in metric space theory. Subsection 5.6 deals with the case of separable Banach spaces, and differs from all other subsections in that we need to build a new and more manageable reduction of linear isometry (restricted to some suitable and abstractly defined class of separable Banach spaces) to an orbit equivalence relation (namely, to an isomorphism relation). Finally, subsection 5.7 contains applications of our main result to all remaining examples of complete analytic quasi-orders (in this case we sketch the proofs of the results as, contrary to all other subsections, they do not involve any genuinely new idea, but are combinations of techniques which already appeared in previous applications).

The following notation, originating from [FS89, will be used several times. For each $n \in \omega$, let $T Y_{n}$ be the set of quantifier free types for the first $n$ variables in $\mathcal{L}$ (an empty 0-type is also considered here), and let $T Y=\bigcup_{n \in \omega} T Y_{n}$. Fix a bijection $e: \omega \rightarrow T Y$ such that $e(i) \in T Y_{n}, e(j) \in T Y_{m}, n<m$ imply $i<j$. (This is possible as $\mathcal{L}$ consists of just one relation symbol.) Each $i \in \omega$ is the code of type $e(i)$. For $G$ an $\mathcal{L}$-structure on $\omega$ and $t \in{ }^{<\omega} \omega$, let $\tau_{G}(t) \in \omega$ be the code of the quantifier free type of $t$ in $G$.

5.1. Epimorphisms between graphs. Given graphs $H, H^{\prime}$ on $\omega$, define the relation $\preceq_{\text {epi }}$ of being epimorphic image by letting $H \preceq$ epi $H^{\prime}$ if and only if there is a surjection $\gamma: \omega \rightarrow \omega$ such that

$$
n, m \text { are adjacent in } H^{\prime} \Rightarrow \gamma(n), \gamma(m) \text { are adjacent in } H \text {. }
$$

Here we recall the construction of [Cam05a, Theorem 1], which defines a continuous function $G \mapsto G^{*}$ from the class of graphs on $\omega$ to itself reducing the relation of embedding to the relation $\preceq_{\text {epi }}$. We will prove some further properties of this construction, which will be used to show invariant universality of $\preceq_{\text {epi }}$.

Let $\left\{N_{t} \mid t \in{ }^{<\omega} \omega\right\}$ be a partition of $\omega$ into infinite sets. Within each $N_{t}$ fix distinct elements $a^{t}, c_{i}^{t}$, for $i \in \omega$ so that $N_{t} \backslash\left\{a^{t}, c_{i}^{t} \mid i \in \omega\right\}$ is still infinite. For any $t \in{ }^{<\omega} \omega, n \in \omega$, let $L_{t n}$ be a graph on $N_{t}$ whose adjacency relation is defined according to the following clauses:

(1) $N_{t}$ is the disjoint union of two sets $B_{t n}=\left\{a^{t}, b_{1}^{t n}, \ldots, b_{n+2}^{t n}\right\}, C_{t n}=\left\{c_{i}^{t}, d_{i}^{t n} \mid\right.$ $i \in \omega\}$ (where the elements in each list are pairwise distinct);

(2) each of $B_{t n}, C_{t n}$ forms a clique in $L_{t n}$;

(3) in addition to the other elements of $B_{t n}$, vertex $b_{n+2}^{t n}$ is adjacent to all elements of $C_{t n}$. 
Given a graph $G$ on $\omega$, the adjacency relation on the graph $G^{*}$ is defined as follows:

- the adjacency relation on each $N_{t}$ is given by $L_{t \tau_{G}(t)}$;

- for each $t \in{ }^{<\omega} \omega, i \in \omega$, vertices $c_{i}^{t}, a^{t^{\top} i}$ are adjacent.

Lemma 5.1. $G \cong H \Longleftrightarrow G^{*} \cong H^{*}$.

Proof. Let $\varphi: \omega \rightarrow \omega$ be an isomorphism between $G$ and $H$. Then $\varphi$ induces componentwise a bijection $\varphi^{\prime}:{ }^{<\omega} \omega \rightarrow{ }^{<\omega} \omega$ with the property $\forall t \in{ }^{<\omega} \omega\left(\tau_{G}(t)=\right.$ $\left.\tau_{H} \varphi^{\prime}(t)\right)$. An isomorphism $\psi: \omega \rightarrow \omega$ between $G^{*}$ and $H^{*}$ is obtained by setting for every $t \in{ }^{<\omega} \omega$ :

- $\psi\left(a^{t}\right)=a^{\varphi^{\prime}(t)}$;

- $\psi\left(b_{j}^{t \tau_{G}(t)}\right)=b_{j}^{\varphi^{\prime}(t) \tau_{H} \varphi^{\prime}(t)}$, for $j \in\left\{1, \ldots, \tau_{G}(t)+2\right\}$;

- $\psi\left(c_{i}^{t}\right)=c_{\varphi(i)}^{\varphi^{\prime}(t)}, \psi\left(d_{i}^{t \tau_{G}(t)}\right)=d_{i}^{\varphi^{\prime}(t) \tau_{H} \varphi^{\prime}(t)}$, for $i \in \omega$.

Conversely, let $\psi: \omega \rightarrow \omega$ be an isomorphism between $G^{*}$ and $H^{*}$. Observe that $\psi\left(a^{t}\right)=a^{t^{\prime}}$, for $t \neq \emptyset$, and the map $\psi^{\prime}$ sending $t$ into $t^{\prime}$ and $\emptyset$ into itself is a Lipschitz bijection ${ }^{<\omega} \omega \rightarrow{ }^{<\omega} \omega$ such that $\forall t \in{ }^{<\omega} \omega\left(\tau_{G}(t)=\tau_{H} \psi^{\prime}(t)\right)$. Imitating the proof of Cam05a, by a back and forth argument inductively construct $u \in S_{\infty}$ such that $v=\bigcup_{n \in \omega} \psi^{\prime}\left(u\lceil n) \in S_{\infty}, \forall n \in \omega\left(\tau_{G}\left(u\lceil n)=\tau_{H} \psi^{\prime}(u\lceil n))\right.\right.\right.$. Then the bijection $u(n) \mapsto v(n)$ is an isomorphism between $G$ and $H$.

Definition 5.2. Let $G$ be a graph. An automorphism $\varphi$ of $G$ is simple if whenever $\varphi(u) \neq u$, then $u$ belongs to a unique maximal clique of $G$ and $\varphi(u)$ belongs to this same clique.

So for graphs of the form $G^{*}$, an automorphism is simple if and only if it leaves all $a^{t}$ fixed, $t \neq \emptyset$. We remark that such an automorphism fixes the elements of the form $b_{\tau_{G}(t)+2}^{t \tau_{\tau}(t)}$ and $c_{i}^{t}$ too, while it can permutate sets of the forms $\left\{b_{1}^{t \tau_{G}(t)}, \ldots, b_{\tau_{G}(t)+1}^{t \tau_{G}(t)}\right\}$ and $\left\{d_{i}^{t \tau_{G}(t)} \mid i \in \omega\right\}$.

Lemma 5.3. If the only automorphism of $G$ is the identity, then all automorphisms of $G^{*}$ are simple.

Proof. Suppose $\psi: \omega \rightarrow \omega$ is a non-simple automorphism of $G^{*}$. Then there exist distinct elements $t, s \in{ }^{m} \omega$, for some $m>0$, such that $\psi\left(a^{t}\right)=a^{s}$. Notice that if $t\left(n_{0}\right), \ldots, t\left(n_{k}\right)$ are distinct and include all values taken by $t$, then $s\left(n_{0}\right), \ldots, s\left(n_{k}\right)$ are distinct and include all values of $s$. By a back and forth argument, build $u, v \in{ }^{\omega} \omega$ such that $\left(t\left(n_{0}\right), \ldots, t\left(n_{k}\right)\right)^{\curvearrowright} u,\left(s\left(n_{0}\right), \ldots, s\left(n_{k}\right)\right)^{\frown} v \in S_{\infty}$ and, letting $x=t^{\curvearrowleft} u, y=s^{\curlyvee} v$, the relation $\forall n>0\left(\psi\left(a^{x\lceil n}\right)=a^{y\lceil n}\right)$ holds. Since $\forall n \in$ $\omega\left(\tau_{G}\left(x\lceil n)=\tau_{G}(y\lceil n))\right.\right.$, the function $x(n) \mapsto y(n)$ is a non-trivial automorphism of $G$.

Lemma 5.4. Given a graph $G$ on $\omega$, the set $H_{G}$ of simple automorphisms of $G$ is a closed subgroup of $S_{\infty}$.

Proof. The fact that $H_{G}$ is a group uses that if $u \in \omega$ belongs to a unique maximal clique in $G$, then the same holds for $\varphi(u)$ for any automorphism $\varphi$ of $G$.

It is now enough to show that $H_{G}$ is closed in the automorphism group of $G$, since the latter is closed in $S_{\infty}$. Let $\varphi_{n}, \varphi$ be automorphisms of $G$ such that $\lim _{n \rightarrow \infty} \varphi_{n}=\varphi \notin H_{G}$. Let $u \in \omega$ be such that $\varphi(u) \neq u$ where either $u$ belongs to more than one maximal clique of $G$ or $\varphi(u)$ does not belong to the same maximal clique as $u$. Then eventually $\varphi_{n}(u)=\varphi(u)$, so $\varphi_{n} \notin H_{G}$. 
Lemma 5.5. The map $G \mapsto H_{G}$ from the space of graphs on $\omega$ to $G\left(S_{\infty}\right)$, assigning to each graph the group of its simple automorphisms, is Borel.

Proof. It is enough to show that given any finite injective sequence $\left(a_{0}, \ldots, a_{n}\right)$ of natural numbers, the class of graphs having a simple automorphism extending $\left(a_{0}, \ldots, a_{n}\right)$ is Borel. A graph $G$ belongs to this class if and only if it satisfies the following Borel conditions, for all $i, j \in\{0, \ldots, n\}$ :

(1) $i, j$ are adjacent in $G$ if and only if $a_{i}, a_{j}$ are adjacent in $G$;

(2) if $i \neq a_{i}$, then:

- for all distinct $u, v \in \omega$, if $i$ is adjacent in $G$ both to $u, v$, then $u, v$ are adjacent in $G$;

- for all distinct $u, v \in \omega$, if $a_{i}$ is adjacent in $G$ to both $u, v$, then $u, v$ are adjacent in $G$;

- $i, a_{i}$ are adjacent in $G$.

Theorem 5.6. The relation $\preceq$ epi of being epimorphic image on countable graphs is invariantly universal. In view of the Lopez-Escobar theorem, this means that if $R$ is an analytic quasi-order on a standard Borel space $X$, then there is an $\mathcal{L}_{\omega_{1} \omega^{-}}$ sentence $\varphi$ such that $R$ is Borel bireducible with the relation $\preceq_{\text {epi }}$ on $\operatorname{Mod}_{\varphi}$, where each element of $\operatorname{Mod}_{\varphi}$ is a graph.

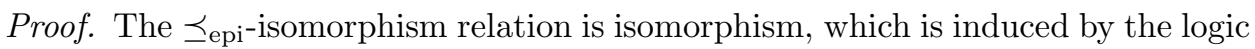
action of $S_{\infty}$ on $\operatorname{Mod}_{\mathcal{L}}$. By Lemma 5.1, the map $G \mapsto G^{*}$ reducing embeddability to $\preceq$ epi also reduces isomorphism to isomorphism. Moreover, by Corollary 3.4 and Lemma 5.3 we get that for $G \in \mathbb{G}$ the group of automorphisms of $G^{*}$ coincides with the group $H_{G}$ of its simple automorphisms, and then by Lemma 5.5 the map $\Sigma$ assigning to each $G \in \mathbb{G}$ the group of automorphisms of $G^{*}$ is Borel. Therefore we can apply Theorem 4.2 to get that $\left(\preceq_{\text {epi }}, \cong\right)$ is invariantly universal.

5.2. Embeddings between colored linear orders. This section and the next deal with various kinds of embeddings for linear orderings.

Denote by $L O$ the Polish space of (strict) linear orderings on $\omega$, and let $R$ be any quasi-order on $\omega$. Define the analytic quasi-order $\preceq_{R}$ on $L O \times{ }^{\omega} \omega$ by letting $(L, c) \preceq_{R}\left(L^{\prime}, c^{\prime}\right)$ if and only if there is an embedding 2 of $L$ into $L^{\prime}$ which preserves $R$, i.e. such that $c(n) R c^{\prime}(g(n))$ for every $n \in \omega$. In [MR04] it was proved that $\preceq=$ is a complete analytic quasi-order, and this result was then extended in Cam05b. using the following construction.

Fix an enumeration $\left\langle k_{n} \mid n \in \omega\right\rangle$ of $\omega$ such that every natural number is listed infinitely many times. In this way, each element $t \in{ }^{<\omega} \omega$ can be seen as a label for the sequence $\lambda_{t}=\left\langle k_{t(0)}, k_{t(1)}, \ldots, k_{t(|t|-1)}\right\rangle \in<\omega \omega$. Given any graph $G$, define the colored order $L_{G}=\left(L_{G}, c_{G}\right)$ by replacing in the lexicographic order of $<\omega \omega \backslash\{\emptyset\}$ each $t$ with an interval $I_{G}^{t}$ (later called a block) of order type $\omega^{2 \tau_{G}\left(\lambda_{t}\right)}$ (this block and its elements will be said to correspond to $t$ or to replace $t$ ), all these points colored by $\tau_{G}\left(\lambda_{t}\right)$. In Cam05b, it was shown that for every $G, G^{\prime}$, if $G$ embeds into $G^{\prime}$, then $L_{G} \preceq=L_{G^{\prime}}$, and if $L_{G} \preceq \geq L_{G^{\prime}}$, then $G$ embeds into $G^{\prime}$ (so that, in particular, any analytic quasi-order $S$ on $L O \times{ }^{\omega} \omega$ such that $\preceq=\subseteq S \subseteq \preceq \geq$ is complete analytic).

\footnotetext{
${ }^{2}$ We can also replace "embedding" with "homomorphism" or "weak-homomorphism", as all these notions coincide on strict linear orderings.
} 
Note that each quasi-order of the form $\preceq_{R}$ is a morphism relation, and both $\preceq=$ and $\preceq \geq$ have the same associated isomorphism relation $\cong_{=}$of color preserving isomorphism. We will now simultaneously show that both $\preceq=$ and $\preceq \geq$ are in fact invariantly universal.

Lemma 5.7. If $L_{G} \cong=L_{G^{\prime}}$ via the isomorphism $g: \omega \rightarrow \omega$, then $G=G^{\prime}$ and $g=i d$.

Proof. Since $g$ must be color preserving and blocks corresponding to sequences of different length are necessarily colored with different colors, we get that the restriction $g_{n}$ of $g$ to any suborder of $L_{G}$ with domain $L_{G}^{n}=\bigcup\left\{I_{G}^{t} \mid t \in{ }^{n} \omega\right\}$ must be an isomorphism between $L_{G}^{n}$ and $L_{G^{\prime}}^{n}$. But any $L_{G}^{n}$ is now a well-order, therefore one inductively gets $\tau_{G}\left(\lambda_{t}\right)=\tau_{G^{\prime}}\left(\lambda_{t}\right)$ for any $t \in{ }^{n} \omega$, which implies that $G=G^{\prime}$ and hence $L_{G}=L_{G^{\prime}}$. Moreover, each $g_{n}$ being defined on a well-order, it must be the identity, hence $g=i d$.

Theorem 5.8. If $S$ is any analytic quasi-order on the space of colored linear orderings on $\omega$ such that $\preceq=\subseteq S \subseteq \preceq_{\geq}$, then $\left(S, \cong_{=}\right)$is invariantly universal. In particular, $\preceq_{=}, \preceq_{\geq}$, as well as any quasi-order of the form $\preceq_{R}$ for $R \subseteq \geq$, are invariantly universal.

Proof. First observe that $\cong=$ is induced by an obvious action of $S_{\infty}$ on $L O \times{ }^{\omega} \omega$ sending $(p,(L, c)) \in S_{\infty} \times\left(L O \times{ }^{\omega} \omega\right)$ into $\left(L^{\prime}, c^{\prime}\right)$, where $L^{\prime}=j_{\mathcal{L}}(p, L)$ and $c^{\prime}(n)=$ $c\left(p^{-1}(n)\right)$. By Lemma [5.7, the map $G \mapsto L_{G}$ reduces $=_{\mathbb{G}}$ to $\cong_{=}$. Finally, since any color preserving automorphism of an $L_{G}$ must be the identity by Lemma 5.7 again, the function $\Sigma$ assigning to each $G \in \mathbb{G}$ the stabilizer of $L_{G}$ is constantly equal to $\{i d\} \in G\left(S_{\infty}\right)$, hence Borel. Now, applying Theorem 4.2, we get the result.

Corollary 5.9. If $R^{\prime}$ is any quasi-order on $\omega$ containing an infinite descending sequence or an infinite anti-chain (i.e. is not a wqo) then $\left(\preceq_{R^{\prime}} \cong_{=)}\right)$is invariantly universal.

Now we want to prove that the relation $\preceq=$, when restricted to colored orderings whose support is fixed to be the order $\mathbb{Q}$, is still invariantly universal (such relation will be denoted by $\preceq_{\mathbb{Q}}$ ). To begin with, we slightly modify a construction from Cam05b, Lemma 3]. Let $L O^{*}$ be the space of linear orderings on $\omega \times \mathbb{Q}$. To each $L=(L, c) \in L O \times{ }^{\omega} \omega$, associate $L^{*}=\left(L^{*}, c^{*}\right) \in L O^{*} \times{ }^{\omega} \times \mathbb{Q}_{\omega}$ in the following way:

- $L^{*}$ is the lexicographic product of $L$ and $\leq$ (so $L^{*}$ is a countable dense linear ordering without endpoints);

- $c^{*}(n, 0)=2 c(n)+1$, and $c^{*}(n, q)=2 \eta(q)$ if $q \neq 0$, where $\eta$ is any bijection between $\mathbb{Q} \backslash\{0\}$ and $\omega$.

As each colored ordering of the type $L^{*}$ can be Borel-in- $L$ identified with a coloring on $\mathbb{Q}$, we can identify $L^{*}$ with its coded version on $\mathbb{Q}$. Now it is easy to check that the Borel map $L \mapsto L^{*}$ is a reduction of $\preceq=$ on $L O \times{ }^{\omega} \omega$ to $\preceq_{\mathbb{Q}}$. For one direction, if $g: \omega \rightarrow \omega$ is an order and color preserving embedding between $L$ and $L^{\prime}$, then the map $(n, q) \mapsto(g(n), q)$ is a witness of $L^{*} \preceq_{\mathbb{Q}}\left(L^{\prime}\right)^{*}$. Conversely, any witness $h$ of $L^{*} \preceq_{\mathbb{Q}}\left(L^{\prime}\right)^{*}$ must be such that $\forall n \exists n^{\prime} h(n, 0)=\left(n^{\prime}, 0\right)$, and this induces an order and color preserving embedding $n \mapsto n^{\prime}$ between $L$ and $L^{\prime}$.

Theorem 5.10. The quasi-order $\preceq_{\mathbb{Q}}$ on $\mathbb{Q}_{\omega}$ is invariantly universal. 
Proof. The $\preceq_{\mathbb{Q}}$-isomorphism relation $\cong_{\mathbb{Q}}$ is induced by the obvious action of the group Aut $(\mathbb{Q})$ of automorphisms of $\mathbb{Q}$. Now consider the map $\mathbb{G} \rightarrow{ }^{\mathbb{Q}} \omega: G \mapsto L_{G}^{*}$, defined as above. We first want to show that this is a reduction of $=_{\mathbb{G}}$ to $\cong_{\mathbb{Q}}$. For the non-trivial direction, if $h$ witnesses $L_{G}^{*} \cong_{\mathbb{Q}} L_{H}^{*}$, then its restriction to $\omega \times\{0\}$ must have range $\omega \times\{0\}$, and hence it induces a witness of $L_{G} \cong=L_{H}$ : but this implies $G=H$ by Lemma 5.7 Finally, we prove that each $L_{G}^{*}$ (where $G \in \mathbb{G}$ ) has no non-trivial color preserving automorphism (so that the map $\Sigma$ assigning to each $G \in \mathbb{G}$ the Aut $(\mathbb{Q})$-stabilizer of $L_{G}^{*}$ is constantly equal to the identity group, hence a Borel map). If $h$ is a color preserving automorphism of $L_{G}^{*}$, then it induces a color preserving automorphism of $L_{G}$ : but since any such isomorphism must be the identity by Lemma 5.7 we must have $h(n, 0)=(n, 0)$ for every $n \in \omega$. Moreover, for every $0 \neq q \in \mathbb{Q}$ and $n \in \omega$ we must have $h(n, q)=\left(n^{\prime}, q\right)$ for some $n^{\prime} \in \mathbb{Q}$, since points of the form $(n, q)$ are the only ones with color $2 \eta(q)$. Finally, since $h(n, 0)=(n, 0)$, we must also have $h(n, q)=(n, q)$ for every $q \in \mathbb{Q}$, because if e.g. $h(n, q)=\left(n^{\prime}, q\right)$ with $q>0$ and $n^{\prime}$ bigger than $n$ in the order $L_{G}$, then there must be some $q^{\prime}$ strictly between 0 and $q$ such that $h\left(n, q^{\prime}\right)=\left(n^{\prime}, 0\right)$, which is clearly impossible as $q^{\prime} \neq 0$. Now we can again apply Theorem 4.2 and get the result.

A linear order is non-scattered if it contains a subset isomorphic to the rationals. We end this section by extending Theorem 5.10 to any countable non-scattered linearly ordered set $L$, that is considering the relation $\preceq_{L}$ of order and color preserving embedding between colorings on $L$. As in Cam05b, Corollary 4], we define a continuous map from ${ }^{\mathbb{Q}} \omega$ into ${ }^{L} \omega$ which assigns to each coloring $c$ on $\mathbb{Q}$ a coloring $\hat{c}$ on $L$ and reduces $\preceq_{\mathbb{Q}}$ to $\preceq_{L}$. First of all, the fact that any non-scattered linear order can be written as a $1+\mathbb{Q}+1$ sum of non-empty orders allows us to write $L$ as $L^{\prime}+\sum_{q \in \mathbb{Q}}\left(U_{q}+\left\{r_{q}\right\}+V_{q}\right)+L^{\prime \prime}$, where $U_{q}$ and $V_{q}$ are non-scattered. For $c \in \mathbb{Q}_{\omega}$ define $\hat{c} \in{ }^{L} \omega$ by letting $\hat{c}(x)=0$ if $x \in L^{\prime} \cup L^{\prime \prime} \cup \bigcup\left\{U_{q}, V_{q} \mid q \in \mathbb{Q}\right\}$, and $\hat{c}\left(r_{q}\right)=c(q)+1$.

Theorem 5.11. If $L$ is a countable non-scattered linear order, the quasi-order $\preceq_{L}$ on ${ }^{L} \omega$ is invariantly universal.

Proof. We will again apply Theorem 4.2 to the map $f: \mathbb{G} \rightarrow{ }^{L} \omega: G \mapsto \hat{L}_{G}^{*}$, hence it is enough to show that the hypotheses of that theorem are satisfied. The $\preceq_{L^{-}}$ isomorphism relation is induced by the natural action of the group $\operatorname{Aut}(L)$ of automorphisms of $L$ on ${ }^{L} \omega$. To show that $f$ reduces $=_{\mathbb{G}}$ to $\cong_{L}$, let $h$ be an isomorphism between $f(G)$ and $f(H)$. Clearly $h\left(r_{q}\right)$ must be of the form $r_{q^{\prime}}$ for some $q^{\prime} \in \mathbb{Q}$, as these are the unique points with non-null color. But then $h$ induces an isomorphism between $L_{G}^{*}$ and $L_{H}^{*}$, which in turn implies $G=H$ by the proof of Theorem 5.10.

It remains to show that the map $\Sigma$ assigning to each $G \in \mathbb{G}$ the $\operatorname{Aut}(L)$-stabilizer of $f(G)=\hat{L}_{G}^{*}$ is Borel. Call each suborder of $L$ of the form $L_{q}=U_{q}+\left\{r_{q}\right\}+V_{q}$ a block (associated to $q$ ), and let $h$ be a color preserving automorphism of $f(G)$. We first want to show that for every $q \in \mathbb{Q}$ there is $q^{\prime} \in \mathbb{Q}$ such that $h\left\lceil L_{q}\right.$ is an order and color preserving isomorphism between $L_{q}$ and $L_{q^{\prime}}$ (from this it follows that $h\left\lceil L^{\prime}\right.$ and $h\left\lceil L^{\prime \prime}\right.$ are order and color preserving automorphisms of $L^{\prime}$ and $L^{\prime \prime}$, respectively). This follows from the fact that each block contains exactly one point with color different from 0 , the image under $h$ of each block (which, in particular, is an interval) must be an interval, and that each interval of $L$ which is not included in a single block (and is included neither in $L^{\prime}$ nor in $L^{\prime \prime}$ ) must contain more than one element with non-null color as the order induced by $L$ on blocks is dense. The 
second step is to show that the image of $L_{q}$ under $h$ must be itself. This is because if $L_{q}$ is sent to $L_{q^{\prime}}$ by $h$, then $h$ induces a color preserving automorphism on $L_{G}^{*}$ sending $q$ to $q^{\prime}$ : but since any color preserving automorphism on $L_{G}^{*}$ must be the identity by the proof of Theorem 5.10, we get $q=q^{\prime}$. By the observations above, $h: L \rightarrow L$ is a color preserving automorphism of $f(G)$ if and only if the following properties hold:

- $h\left\lceil L^{\prime}\right.$ and $h\left\lceil L^{\prime \prime}\right.$ are automorphisms of $L^{\prime}$ and $L^{\prime \prime}$;

- $h\left\lceil U_{q}\right.$ and $h \uparrow V_{q}$ are automorphisms of $U_{q}$ and $V_{q}$ for every $q \in \mathbb{Q}$;

- $h\left(r_{q}\right)=r_{q}$ for every $q \in \mathbb{Q}$.

Since these conditions are independent of $G$, we have that all $f(G)$ have the same fixed stabilizer, so that the map $\Sigma$ is constant (hence, in particular, Borel).

Remark 5.12. As observed in FMR11 about the invariant universality of embeddability between (ordered) graphs, most of the results of this paper have an effective counterpart as well, i.e. the one obtained by systematically replacing "analytic" and "Borel" by (lightface) $\Sigma_{1}^{1}$ and $\Delta_{1}^{1}$ in all definitions, statements and proofs. However, our proof of Theorem 5.11 explicitly uses the countably many parameters $\operatorname{Aut}\left(L^{\prime}\right), \operatorname{Aut}\left(L^{\prime \prime}\right), \operatorname{Aut}\left(U_{q}\right)$, and $\operatorname{Aut}\left(V_{q}\right)$ to show that the stabilizer map is Borel. Therefore this proof does not give a proof of the effective counterpart of Theorem 5.11 (whether such a result holds is still an open problem), but just of the effective counterpart relativized to those parameters. A similar remark also holds for Theorem 5.14 below.

5.3. Dense order preserving embeddings. Following [MR04, a function $g: \mathbb{Q} \rightarrow \mathbb{Q}$ is dense order preserving if it is increasing and for all $q_{0}, q_{1}, r_{0}, r_{1} \in \mathbb{Q}$ with $f\left(q_{0}\right)<r_{0}<r_{1}<f\left(q_{1}\right)$ there exists $q \in \mathbb{Q}$ such that $r_{0}<f(q)<r_{1}$. This means that range $(g)$ is dense in the (real) interval (inf range $(g)$, sup range $(g)$ ). Moreover, in MR04 the dense order preserving functions were characterized as the restrictions to $\mathbb{Q}$ of those continuous increasing functions $g$ from the real line $\mathbb{R}$ into itself such that $g(\mathbb{Q}) \subseteq \mathbb{Q}$.

Given a quasi-order $R$ on an at most countable set $C$, consider the analytic quasi-order $\leq_{\text {dop }}^{R}$ on the Polish space ${ }^{\mathbb{Q}} C$ of $C$-colorings of $\mathbb{Q}$ defined by $c \leq_{\text {dop }}^{R} c^{\prime}$ if and only if there is a dense order preserving function $g: \mathbb{Q} \rightarrow \mathbb{Q}$ such that $c(q) R c^{\prime}(g(q))$ for every $q \in \mathbb{Q}$. Extending previous results of [MR04, it was proved in Cam05b that when $R$ is one of the relations $=$ on $\omega, \geq$ on $\omega$, or $=$ on 2 (which for simplicity of notation will be denoted in the sequel by $=, \geq$ and $=_{2}$, respectively) the corresponding quasi-order $\leq_{\text {dop }}^{R}$ becomes complete analytic. In this section we want to further extend such results by showing that these quasi-orders are in fact invariantly universal.

The case of $=_{2}$ implies that of $=$ but, as in Cam05b, dealing with $=$ and $\geq$ simultaneously will provide results for all analytic quasi-orders pinched between $\leq_{\text {dop }}$ and $\leq_{\text {dop }}^{\geq}$. Fix partitions $\left\{Q, Q_{n} \mid n \in \omega\right\}$ and $\left\{P_{n} \mid n \in \omega\right\}$ of $\mathbb{Q}$ into dense subsets, and for $\nu \in{ }^{<\omega} \omega \backslash\{\emptyset\}$, let $Q_{\nu}=Q_{\nu(0)} \times \cdots \times Q_{\nu(|\nu|-1)}$. If $t \in{ }^{l+1} \mathbb{Q}$, we call $t$ good if there is $\nu \in{ }^{l+1} \omega$ such that $t \in Q_{\nu}$ (for any good $t$ such a $\nu$ is clearly unique and will be denoted by $\nu_{t}$ ), and bad if there is $i \leq l$ such that $t(i) \in Q$. Also, for $n \geq 1$ let $W_{n}=\{1,2, \ldots, n\}$. Finally, let $A=\left({ }^{<\omega} \mathbb{Q} \backslash\{\emptyset\}\right) \times \mathbb{Q}$ be endowed with the lexicographic order (where $<\omega \mathbb{Q}$ is ordered lexicographically and $\mathbb{Q}$ is ordered by $\leq$, so that $A$ is isomorphic to $\mathbb{Q}$ and we can identify them), and for $t \in{ }^{<\omega} \mathbb{Q} \backslash\{\emptyset\}$ let $J_{t}=\{t\} \times \mathbb{Q}$. We will now construct a continuous map $G \mapsto G^{+}$from the space of 
graphs on $\omega$ to the space ${ }^{A} \omega$ of colorings on $A$. Let $G$ be given, and let $(t, q) \in A$ with $|t|=l+1$, some $l \in \omega$. Let $\alpha(n)$ denote the least $i$ such that $e(i) \in T Y_{n}$. If $t$ is good, then put:

$$
\begin{aligned}
& \text { - } G^{+}(t, q)=0 \text { if } q \in W_{\tau_{G}\left(\nu_{t}\right)+l} \text { and } \\
& \text { - } G^{+}(t, q)=\tau_{G}\left(\nu_{t}\right)+l+n \text { if } q \in P_{n} \backslash W_{\tau_{G}\left(\nu_{t}\right)+l} .
\end{aligned}
$$

If on the other hand $t$ is bad, then set

$$
\begin{aligned}
& \text { - } G^{+}(t, q)=0 \text { if } q \in W_{\alpha(l+1)+l} \text { and } \\
& \text { - } G^{+}(t, q)=\alpha(l+2)+l+n \text { if } q \in P_{n} \backslash W_{\alpha(l+1)+l} .
\end{aligned}
$$

In Cam05b, Theorem 6], it was already proved that if $G$ embeds into $H$, then $G^{+} \leq_{\text {dop }}^{=} H^{+}$, while if $G^{+} \leq_{\text {dop }}^{\geq} H^{+}$, then $G$ embeds into $H$. This in particular implies that the map $G \mapsto G^{+}$is a reduction of embeddability between graphs on $\omega$ to both $\leq \leq_{\text {dop }}$ and $\leq \underset{\text { dop }}{\geq}$

Theorem 5.13. The quasi-orders $\leq_{\mathrm{dop}}^{=}$and $\leq_{\mathrm{dop}}^{\geq}$are invariantly universal. More generally, if $S$ is any analytic quasi-order on $\mathbb{Q}_{\omega}$ such that $\leq \overline{\bar{d}}_{\mathrm{dop}} \subseteq S \subseteq \leq_{\mathrm{dop}}$, then $\left(S, \cong{ }_{\mathrm{dop}}\right)$ (where $c \cong{ }_{\mathrm{dop}} c^{\prime}$ if and only if there is a color preserving automorphism of $\mathbb{Q})$ is invariantly universal.

Proof. Identify $\mathbb{Q}$ with $A$. In order to apply Theorem 4.2 , first notice that $\cong_{\text {dop }}$ is the isomorphism relation associated to both $\leq_{\text {dop }}$ and $\leq_{\text {dop }}^{\geq}$, and that it is induced by the group $\operatorname{Aut}(\mathbb{Q})$ of automorphisms of $\mathbb{Q}$ (note that any automorphism of $\mathbb{Q}$ is automatically a dense order preserving function since it is surjective). Now consider the map $G \mapsto G^{+}$previously defined. If $h: A \rightarrow A$ is a color and (dense) order preserving isomorphism of $G^{+}$to $H^{+}$, the following hold (see the proof of Cam05b, Theorem 6] for more details):

i) $\forall t \in<\omega \mathbb{Q} \backslash\{\emptyset\} \exists s \in<\omega \mathbb{Q} \backslash\{\emptyset\}\left(h\left(J_{t}\right)=J_{s}\right)$;

ii) defining $\rho(t)=s$ if $h\left(J_{t}\right)=J_{s}, \rho$ is an order and length preserving bijection;

iii) $t$ is good if and only if $\rho(t)$ is good, and in this case $\tau_{G}\left(\nu_{t}\right)=\tau_{H}\left(\nu_{\rho(t)}\right)$.

Using these facts as at the end of the proof of Cam05b, Theorem 6], by a back and forth argument we have that $G^{+} \cong$ dop $H^{+}$implies $G \cong H$. Since for $G, H \in \mathbb{G}$ we have $G \cong H \Longleftrightarrow G=H \Longleftrightarrow G^{+}=H^{+}$we get that $G \cong H \Longleftrightarrow G^{+} \cong{ }_{\text {dop }} H^{+}$.

It remains to prove that the map $\Sigma$ associating to each $G \in \mathbb{G}$ the $\operatorname{Aut}(\mathbb{Q})$ stabilizer of $G^{+}$is Borel. By an argument similar to that of Lemma 5.3, one can check that since $G$ has no non-trivial automorphisms, then if $h$ is a color preserving automorphism of $G^{+}$and $\rho$ is defined as in ii) above, $t=\rho(s)$ and $t$ good imply $\nu_{t}=\nu_{s}$, which in turn implies that $(t, q)$ and $(s, q)$ have same color for every $q \in \mathbb{Q}$; the same trivially holds in the case when $t$ is bad, as by iii) $t$ is good if and only if $\rho(t)$ is good. Therefore one can check that given a map $\varphi: B \rightarrow A$ (where $B$ is a finite subset of $A), G^{+}$has a color preserving automorphism extending $\varphi$ if and only if the conjunction of the following Borel conditions is satisfied:

- $\varphi$ is an order and color preserving injection;

- for every $b \in B$, if $t, s \in{ }^{<\omega} \mathbb{Q} \backslash\{\emptyset\}$ are such that $b \in J_{t}$ and $\varphi(b) \in J_{s}$, then $-|t|=|s|$,

$-t$ is good if and only if $s$ is good, and if $t$ is good, then $\nu_{t}=\nu_{s}$;

- for every $b, b^{\prime} \in B$ and $t, s \in{ }^{\omega} \mathbb{Q} \backslash\{\emptyset\}$, if $b, b^{\prime} \in J_{t}$, then $\varphi(b) \in J_{s} \Longleftrightarrow$ $\varphi\left(b^{\prime}\right) \in J_{s}$; 
- for every $b \in B$, if $b \in J_{t}$ with $t$ bad, $\varphi(b) \in J_{s}$ and $0<m<|t|$, setting $r=t\lceil m, u=s\lceil m$, one has

$-r$ is good $\Longleftrightarrow u$ is good,

- if $r$ is good, then $\nu_{r}=\nu_{u}$;

- for every $b, b^{\prime} \in B$, if $b \in J_{t}, b^{\prime} \in J_{t^{\prime}}, \varphi(b) \in J_{s}, \varphi\left(b^{\prime}\right) \in J_{s^{\prime}}$ then $t \subseteq t^{\prime} \Longleftrightarrow$ $s \subseteq s^{\prime}$

- for every $t \in{ }^{<\omega} \mathbb{Q} \backslash\{\emptyset\}$, for every $1 \leq i<k_{t}$ (where $k_{t}$ is either $\tau_{G}\left(\nu_{t}\right)+l$ or $\alpha(l+1)+l$, depending on whether $t$ is good or bad), and for every $(t, q) \in B$, if $\varphi(t, q)=\left(s, q^{\prime}\right)$, then

a) $q<1 \Longleftrightarrow q^{\prime}<1$,

b) $i<q<i+1 \Longleftrightarrow i<q^{\prime}<i+1$,

c) $q>k_{t} \Longleftrightarrow q^{\prime}>k_{t}$,

d) $q=i \Longleftrightarrow q^{\prime}=i$ and $q=k_{t} \Longleftrightarrow q^{\prime}=k_{t}$.

We end this section with the proof that the quasi-order $\leq_{\text {dop }}^{=2}$ on the space ${ }^{\mathbb{Q}} 2$ of two-color colorings of $\mathbb{Q}$ is already invariantly universal. We first recall the following construction from [Cam05b, Theorem 8]. Let $\left\{L_{n} \mid n \in \omega\right\}$ be a collection of linear orders such that no one of them is isomorphic to an interval of another, and for each $l \in \omega$ fix scattered subsets $W_{l}^{\alpha(l+1)}, W_{l}^{\alpha(l+1)+1}, \ldots, W_{l}^{\alpha(l+2)-1}$ of $\mathbb{Q}$ pairwise incomparable under embeddability. For $t \in<\omega \mathbb{Q} \backslash\{\emptyset\}$, set $J_{t}=L_{|t|-1} \times 2 \times \mathbb{Q}$, and let $A=\left\{(t, u, i, r) \mid t \in{ }^{<\omega} \mathbb{Q} \backslash\{\emptyset\},(u, i, r) \in J_{t}\right\}$ be endowed with the lexicographic order, so that $A$ is isomorphic to $\mathbb{Q}$. Define $t$ to be good or bad as done before. Given a countable graph $G$, define $G^{\prime}$ to be the coloring on $A$ defined by letting $(t, u, i, r)$ to have color $i$ if and only if:

- either $t$ is bad,

- or else $t$ is good and either $i=0$ or $r \notin W_{|t|-1}^{\tau_{G}\left(\nu_{t}\right)}$.

Otherwise (that is if $t$ is good, $i=1$ and $r \in W_{|t|-1}^{\tau_{G}\left(\nu_{t}\right)}$ ) let the color be 0. In Cam05b, Theorem 8] it is proved that the continuous map $G \mapsto G^{\prime}$ reduces embeddability between countable graphs to $\leq_{\text {dop }}^{=2}$.

Theorem 5.14. The quasi-order $\leq_{\mathrm{dop}}^{=2}$ is invariantly universal. Therefore, if $R$ is a quasi-order on $\omega$ with at least two incomparable elements, then $\left(\leq_{\mathrm{dop}}^{R}, \cong_{\mathrm{dop}}\right)$ is invariantly universal.

Proof. The application of Theorem 4.2 will suffice again, so we just have to show that the hypotheses of that theorem are satisfied. Identify $A$ with $\mathbb{Q}$. Clearly, the isomorphism relation associated to $\leq_{\text {dop }}^{=2}$ is $\cong$ dop again, so it is induced by the natural action of $\operatorname{Aut}(\mathbb{Q})$. That the map $G \mapsto G^{\prime}$ reduces $\cong_{\mathbb{G}}$ to $\cong_{\text {dop }}$ essentially follows from the fact that properties similar to conditions i)-iii) given in the proof of Theorem 5.13 hold also with respect to our new construction, so we just need to prove that the map $\Sigma$ sending each $G \in \mathbb{G}$ to the $\operatorname{Aut}(\mathbb{Q})$-stabilizer of $G^{\prime}$ is Borel. Arguing as before, since $G$ has no non-trivial automorphisms, then if $h$ is a color preserving automorphism of $G^{\prime}$ and $\rho$ is defined as in ii) above, $s=\rho(t)$ and $t$ good imply $\nu_{t}=\nu_{s}$, which in turn implies that $(t, u, i, r)$ and $(s, u, i, r)$ have same color for every $(u, i, r) \in L_{|t|-1} \times 2 \times \mathbb{Q}$ (the same is trivially true for bad $t$ and $s$ again). Moreover, it is not hard to check that for every $(t, u, i, r),\left(t, u^{\prime}, i^{\prime}, r^{\prime}\right) \in A$ if $h(t, u, i, r)=(s, v, j, p)$ and $h\left(t, u^{\prime}, i^{\prime}, r^{\prime}\right)=\left(s, v^{\prime}, j^{\prime}, r^{\prime}\right)$, then $i=j$ (which in particular implies $i=i^{\prime} \Rightarrow j=j^{\prime}$ ) and $u=u^{\prime} \Rightarrow v=v^{\prime}$. Therefore we have that given $\varphi: B \rightarrow A$, where $B$ is a finite subset of $A, G^{\prime}$ has a color preserving 
automorphism extending $\varphi$ if and only if the conjunction of the following Borel conditions is satisfied:

- $\varphi$ is an order and color preserving injection;

- if $(t, u, i, r),\left(t^{\prime}, u^{\prime}, i^{\prime}, r^{\prime}\right) \in B, \varphi(t, u, i, r)=(s, v, j, p)$ and $\varphi\left(t^{\prime}, u^{\prime}, i^{\prime}, r^{\prime}\right)=$ $\left(s^{\prime}, u^{\prime}, i^{\prime}, p^{\prime}\right)$, then

$-|t|=|s|$,

$-t$ is good if and only if $s$ is,

- if $t$ is good, then $\nu_{t}=\nu_{s}$,

$-i=j$,

$-t=t^{\prime} \Rightarrow s=s^{\prime}$,

$-t=t^{\prime} \wedge u=u^{\prime} \Rightarrow s=s^{\prime} \wedge v=v^{\prime}$

- for every $(t, u, i, r) \in B$ with $t$ bad, if $\varphi(t, u, i, r)=(s, v, i, p), 0<m<$ $|t|, t^{\prime}=t\left\lceil m, s^{\prime}=s\lceil m\right.$, then

$-t^{\prime}$ is good $\Longleftrightarrow s^{\prime}$ is good,

- if $t^{\prime}$ is good, then $\nu_{t^{\prime}}=\nu_{s^{\prime}}$;

- if $(t, u, i, r),\left(t^{\prime}, u^{\prime}, i^{\prime}, r^{\prime}\right) \in B, \varphi(t, u, i, r)=(s, v, i, p), \varphi\left(t^{\prime}, u^{\prime}, i^{\prime}, r^{\prime}\right)=$ $\left(s^{\prime}, v^{\prime}, i^{\prime}, p^{\prime}\right)$, then $t \subseteq t^{\prime} \Longleftrightarrow s \subseteq s^{\prime}$

- for each $t \in{ }^{l+1} \mathbb{Q}, \varphi_{t}$ : $\operatorname{dom} \varphi_{t} \subseteq L_{l} \rightarrow L_{l}$ can be extended to an automorphism of $L_{l}$, where $\varphi_{t}$ is defined on $u$ with value $v$ if there is $(t, u, i, r) \in B$ such that $\varphi(t, u, i, r)$ is of the form $(s, v, i, p)$ for some $s, p$ ( $\varphi_{t}$ is well defined when the previous conditions are satisfied);

- for each $t \in{ }^{l+1} \mathbb{Q}, u \in L_{l}$, the map $\varphi_{t, u}: \mathbb{Q} \rightarrow \mathbb{Q}$ can be extended to an automorphism of $\mathbb{Q}$ which setwise fixes $W_{t}$, where $\varphi_{t, u}(r)=p$ if there is $(t, u, 1, r) \in B$ such that $\varphi(t, u, 1, r)=(s, v, 1, p)$ for some $s, v, W_{t}=\emptyset$ if $t$ is bad, and $W_{t}=W_{l}^{\tau_{G}\left(\nu_{t}\right)}$ if $t$ is good.

5.4. Continuous embeddings between compacta. In this section, Theorem 4.2 will be applied to show the invariant universality of the pair $\left(\sqsubseteq_{c}, \simeq\right)$, where $\sqsubseteq_{c}$ is the relation of continuous embeddability between dendrites (that is, compact, connected, locally connected metric spaces not containing circles) and $\simeq$ is homeomorphism. Dendrites form a standard Borel space, in fact a $\boldsymbol{\Pi}_{3}^{0}$-complete subset of the hyperspace $K\left({ }^{\omega}[0,1]\right)$, by results in CDM05. If a dendrite is the union of finitely many arcs, then it is called a tree. The order $\operatorname{ord}(p, X)$ of a point $p$ in a topological space $X$ is the smallest cardinal $\kappa$ such that $p$ has an open neighborhood basis in $X$ whose members have boundaries of cardinality at most $\kappa$.

For a point $p$ in a dendrite $D$, the inequality $\operatorname{ord}(p, D) \leq \omega$ holds. If $\operatorname{ord}(p, D)=$ 1 , then $p$ is called an end point of $D$; if $\operatorname{ord}(p, D) \geq 3$, then it is called a branch point. A maximal open free arc in $D$ is a subset of $D$ homeomorphic to $(0,1)$ that does not contain branch points of $D$ and is maximal with these properties. What we shall actually show is the invariant universality restricted to the class $Z$ of dendrites having infinitely many maximal open free arcs, infinitely many branch points and whose points have order at most 3 (the fact that this is a Borel set can be recovered from the proofs of [CDM05, Lemma 6.5] and of [MR04, Lemma 1.4]). Again by CDM05, the set of pairs $(D, p) \in Z \times{ }^{\omega}[0,1]$ such that $p$ is a branch point of $D$ is a Borel set (with countable vertical sections) and the same for the set of $(D, A) \in Z \times K\left({ }^{\omega}[0,1]\right)$ such that $A$ is the closure of a maximal open free arc in $D$. 
As discussed after Theorem 4.2, we now need a map $f$ from countable graphs to $Z$ and a map $g$ reducing $\simeq$ to a suitable orbit equivalence relation. Function $f$ has already been defined in Cam05b, and we shall recall its definition here; its range will actually yield dendrites contained in the square ${ }^{2}[0,1]$. As for $g$, we shall partially modify a construction of [CDM05], since this will make it easier to check the properties we need.

First we define the function $f$ and prove that it has the desired properties. Fix trees $T_{0}, T_{1}, T_{2} \in Z$ with the property that

- the order of points in each $T_{j}$ is at most 3 ;

- each $T_{j}$ has an end point $p_{j}$ such that for $i \neq j$ there is no continuous embedding $\varphi: T_{i} \rightarrow T_{j}$ with $\varphi\left(p_{i}\right)=p_{j}$.

Also fix an enumeration $\left\langle q_{n} \mid n \in \omega\right\rangle$ of $\mathbb{Q} \cap\left(\frac{1}{2}, 1\right)$ and an increasing bijection $h: \mathbb{Q} \cap$ $\left(\frac{1}{2}, 1\right) \rightarrow \mathbb{Q}$. For $(n, j) \in \omega \times 2$, let $g_{n j}: T_{j} \rightarrow\left[\frac{1}{2}, 1\right] \times[0,1]$ be a continuous embedding whose range $T_{n}^{j}$ has diameter less than $\frac{1}{n+1}$ and such that $g_{n j}\left(p_{j}\right)=\left(q_{n}, 0\right)$ and $T_{n}^{j} \cap T_{n^{\prime}}^{j^{\prime}}=\emptyset$ for $n \neq n^{\prime}$. Similarly, fix a continuous embedding $\bar{g}: T_{2} \rightarrow\left[0, \frac{1}{2}\right) \times[0,1]$ with $\bar{g}\left(p_{2}\right)=\left(\frac{1}{4}, 0\right)$ and let $T$ be its range.

Now, given a graph $G$ on $\omega$, let $G^{\prime}$ be as in Theorem 5.14 and let

$$
f(G)=([0,1] \times\{0\}) \cup T \cup \bigcup_{n \in \omega} T_{n}^{\gamma_{n}},
$$

where $\gamma_{n} \in 2$ is the color assigned by $G^{\prime}$ to $h\left(q_{n}\right)$. Notice that $f(G) \in Z$.

The fact that $f$ reduces embeddability of graphs on $\omega$ to $\sqsubseteq_{c}$ is contained in Cam05b. Suppose that $f(G) \simeq f(H)$, for $G, H \in \mathbb{G}$; then $G^{\prime} \cong_{\text {dop }} H^{\prime}$ and $G=H$ by the previous section.

Now we define the function $g$. Let $\Lambda=\{P, R\}$, where $P$ is a unary relation symbol and $R$ is a ternary relation symbol. In CDM05] a function $\Phi$ is built reducing homeomorphism on $Z$ (actually on a bigger class) to isomorphism in $\operatorname{Mod}_{\Lambda}$, the Polish space of $\Lambda$-structures on $\omega$ : roughly speaking, given $D \in Z$ the natural numbers (which constitute the domain of $\Phi(D)$ ) are used as codes for the branching points and the closure of the maximal open free $\operatorname{arcs}$ of $D$, the interpretation in $\Phi(D)$ of the predicate $P$ identify the (codes for) branching points of $D$, and the ternary relation $R$ is interpreted in $\Phi(D)$ as the relation of "being in between". Here we use a similar reduction $g: Z \rightarrow \operatorname{Mod}_{\Lambda}$ by taking $g \nmid(Z \backslash f(\mathbb{G}))=\Phi \uparrow(Z \backslash f(\mathbb{G}))$ and defining $g$ on $f(\mathbb{G})$ in such a way to keep $g(D)$ isomorphic with $\Phi(D)$, so to grant that $g$ will still be a reduction.

Let $\beta_{n}: f(\mathbb{G}) \rightarrow{ }^{2}[0,1]$ be a sequence of Borel functions such that $\beta_{n}(D)$ enumerate the branch points of $D$ having positive second coordinate, so that the set of branch points of $D$ is $\left\{\left(\frac{1}{4}, 0\right)\right\} \cup\left(\left(\mathbb{Q} \cap\left(\frac{1}{2}, 1\right)\right) \times\{0\}\right) \cup\left\{\beta_{n}(D) \mid n \in \omega\right\}$. Similarly, let $\alpha_{n}: f(\mathbb{G}) \rightarrow K\left({ }^{2}[0,1]\right)$ be a sequence of Borel functions such that $\alpha_{n}(D)$ enumerate the closures of the maximal open free arcs of $D$ not lying on $[0,1] \times\{0\}$. The collection of closures of maximal open free $\operatorname{arcs}$ of $D$ is thus $\left\{\left[0, \frac{1}{4}\right] \times\{0\},\left[\frac{1}{4}, \frac{1}{2}\right] \times\{0\}\right\} \cup\left\{\alpha_{n}(D) \mid n \in \omega\right\}$. Now let $p_{n}: f(\mathbb{G}) \rightarrow K\left({ }^{2}[0,1]\right)$ be 
defined by letting

$$
p_{n}(D)=\left\{\begin{array}{lll}
\left\{\left(\frac{1}{4}, 0\right)\right\} & \text { if } & n=0, \\
\left\{\beta_{m}(D)\right\} & \text { if } & n=4 m+2, \\
\left\{\left(q_{m}, 0\right)\right\} & \text { if } & n=4 m+4, \\
{\left[0, \frac{1}{4}\right] \times\{0\}} & \text { if } & n=1, \\
{\left[\frac{1}{4}, \frac{1}{2}\right] \times\{0\}} & \text { if } & n=3, \\
\alpha_{m}(D) & \text { if } & n=2 m+5 .
\end{array}\right.
$$

So $\left\{p_{n}(D) \mid n \in \omega\right\}$ is an enumeration of branch points and closures of maximal open free $\operatorname{arcs}$ of $D$.

To define function $g$ on $f(\mathbb{G})$ consider $D \in f(\mathbb{G})$. For $n, m, m^{\prime}, m^{\prime \prime} \in \omega$, let

- $P^{g(D)}(n)$ if and only if $n$ is even,

- $R^{g(D)}\left(m, m^{\prime}, m^{\prime \prime}\right)$ if and only if all points in $p_{m^{\prime}}(D)$ lie on any path from a point in $p_{m}(D)$ to a point in $p_{m^{\prime \prime}}(D)$.

As in the proof of [CDM05, Lemma 6.5], function $g$ is Borel (since $f(\mathbb{G})$ is Borel by the injectivity of $f$ ) and reduces the homeomorphism relation on $Z$ to the isomorphism relation on $\operatorname{Mod}_{\Lambda}$.

Theorem 5.15. The pair $\left(\complement_{c}, \simeq\right)$ on dendrites is invariantly universal: for any analytic quasi-order $R$ there is a Borel class $\mathcal{C}$ of dendrites closed under homeomorphism such that $R$ is Borel bireducible with the restriction of $\bigsqcup_{c}$ to $\mathcal{C}$.

Proof. For $l \neq 1,3$ let $\kappa_{l}: f(\mathbb{G}) \rightarrow\{4 m \mid m \in \omega\}$ be defined by setting $\kappa_{l}(D)=n$ if and only if $p_{n}(D)$ is the value that the first point map on $[0,1] \times\{0\}$ assumes on some (all) points of $p_{l}(D)$. Notice the following facts:

(1) Functions $\kappa_{l}$ are Borel: indeed, $\kappa_{l}(D)=n \Leftrightarrow \neg \exists r \in\{4 m \mid m \in \omega\}(r \neq$ $\left.n \wedge R^{g(D)}(l, r, n)\right)$.

(2) Denoting for $D \in f(\mathbb{G}), n \in \omega$ by $L_{D}(n) \in 2$ the color assigned by $\left(f^{-1}(D)\right)^{\prime}$ to $h\left(q_{n}\right)$, the map $f(\mathbb{G}) \rightarrow{ }^{\omega} 2: D \mapsto L_{D}$ is Borel.

By the observations preceding this theorem, in order to apply Theorem 4.2 it remains only to show that the map $\Sigma^{\prime}: f(\mathbb{G}) \rightarrow G\left(S_{\infty}\right)$ assigning to each $D \in f(\mathbb{G})$ the stabilizer $\operatorname{Stab}(g(D))$ of $g(D)$ is Borel (so that the map $\Sigma$ assigning $\operatorname{Stab}(g(f(G)))$ to each $G \in \mathbb{G}$, which is simply the composition of $f$ and $\Sigma^{\prime}$, is Borel as well). So fix a finite injective sequence $s=\left(s_{0}, \ldots, s_{k}\right)$ with $k \geq 3$ of natural numbers, in order to check that

$$
V_{s}=\{D \in f(\mathbb{G}) \mid \exists \varphi \in \operatorname{Stab}(g(D))(s \subseteq \varphi)\}
$$

is Borel. Indeed, $D \in V_{s}$ is equivalent to the following Borel conditions on $D$ :

- $s_{0}=0, s_{1}=1, s_{2}=2$;

- $P^{g(D)}(i) \Leftrightarrow P^{g(D)}(s(i))$, for $i \in\{0, \ldots, k\}$

- $R^{g(D)}\left(i, i^{\prime}, i^{\prime \prime}\right) \Leftrightarrow R^{g(D)}\left(s_{i}, s_{i^{\prime}}, s_{i^{\prime \prime}}\right)$, for $i, i^{\prime}, i^{\prime \prime} \in\{0, \ldots, k\}$;

- $\kappa_{i}(D)=\kappa_{i^{\prime}}(D) \Leftrightarrow \kappa_{s_{i}}(D)=\kappa_{s_{i^{\prime}}}(D)$, for $i, i^{\prime} \in\{0, \ldots, k\} \backslash\{1,3\}$;

- for any $n$ such that $\kappa_{\bar{l}}(D)=n$ for some $\bar{l} \leq k$, the restriction of $s$ to $\left\{l \leq k \mid \kappa_{l}(D)=n\right\}$ is extendible to an isomorphism $\psi$ between the finite structures $\mathcal{A}=\left\{l \in \omega \mid \kappa_{l}(D)=n\right\}$ and $\mathcal{B}=\left\{l \in \omega \mid \kappa_{l}(D)=\kappa_{s_{\bar{l}}}(D)\right\}$;

- there is an automorphism of $g(D)$ extending the function sending $\kappa_{l}(D)$ to $\kappa_{s_{l}}(D)$ for all $l \in\{0, \ldots, k\} \backslash\{1,3\}$.

This last set is Borel, since it is the set of all $D \in f(\mathbb{G})$ such that there is a color preserving automorphism of $\left(f^{-1}(D)\right)^{\prime}$ extending the function that sends each 
$h\left(q_{\frac{\kappa_{l}(D)-4}{4}}\right)$ to $h\left(q_{\frac{\kappa_{s_{l}}(D)-4}{4}}\right)$ when $\kappa_{l}(D) \neq 0$; thus this follows from the proof of Theorem 5.14

5.5. Isometric embeddings between Polish metric spaces. Now we consider the case of isometric embeddability on (some classes of) Polish metric spaces. Recall that $(X, d)$ is a Polish metric space if $d$ is a complete metric on $X$ which generates a second countable topology on $X$. It is a well-known fact that there is a universal Polish space $\mathbb{U}$ (called Urysohn space) such that each Polish metric space is isometric to a closed subspace of $\mathbb{U}$ and $\mathbb{U}$ is ultrahomogeneous (such a $\mathbb{U}$ is unique up to isometry). Therefore it is natural to consider the space $F(\mathbb{U})$ of the closed subspaces of $\mathbb{U}$ (endowed with the usual Effros Borel structure, see [Kec95, §12.C]) as the standard Borel space of Polish metric spaces. As shown in [LR05, the relation of isometric embeddability $\complement^{i}$ (that is the quasi-order on $F(\mathbb{U})$ induced by isometric embeddings, i.e. distance preserving functions, between Polish metric spaces) is a complete analytic quasi-order ( $a n d \cong^{i}$, the relation of being isometric, is clearly the $\sqsubseteq^{i}$-isomorphism). To be more precise, Louveau and Rosendal gave two different proofs of the completeness of $\sqsubseteq^{i}$ by considering two specific subclasses of $F(\mathbb{U})$ : the class of discrete Polish metric spaces (i.e. spaces in which the induced topology is discrete) and ultrametric Polish spaces (i.e. spaces in which the metric $d$ is actually an ultrametric, that is $d$ satisfies the inequality $d(x, z) \leq \max \{d(x, y), d(y, z)\}$ for all points $x, y, z$ of the space). It is natural to ask whether $\sqsubseteq^{i}$ is invariantly universal, and even whether its restrictions to the subclasses above are invariantly universal (such restricted versions easily imply the general result, as the notions of discrete Polish metric space and ultrametric Polish space are invariant under isometry).

We first consider the easier case of discrete Polish metric spaces. Note that this class was also considered in FMR11, although with a different coding: in that paper the class of discrete Polish metric spaces was defined as the collection of the spaces of the form $(\omega, d)$ where $d$ induces a discrete topology on $\omega$ (this can be done since any discrete Polish metric space must be countable), while here we want to consider the class $\mathcal{D} \subseteq F(\mathbb{U})$ of those infinite spaces $(X, d)$ such that $d$ induces a discrete topology, which is potentially a much harder problem. Let us note here that $\mathcal{D}$ is not a standard Borel space as it is a proper co-analytic subset of $F(\mathbb{U})$; see [Kec95, Exercise 27.8]. In fact, we shall prove the result for a Borel $Z \subseteq \mathcal{D}$ invariant under isometry.

Given a combinatorial tree $G$ on $\omega$, consider the discrete Polish metric space $D_{G}$ whose domain is $\omega$ and whose distance $d_{G}$ is the geodesic distance on $G$, that is $d_{G}(n, m)=$ the length of the (unique) path joining $n$ to $m$ in $G$. Such a $D_{G}$ will then be Borel-in- $G$ coded as an element of $\mathcal{D} \subseteq F(\mathbb{U})$, also denoted by $D_{G}$. As noted in LR05 and FMR11, from such a $D_{G}$ one can recover the structure of $G$ by linking two vertices $n, m$ if and only if $d_{G}(n, m)=1$, so that we clearly have $G \cong H \Longleftrightarrow D_{G} \cong^{i} D_{H}$ and $G \sqsubseteq H \Longleftrightarrow D_{G} \sqsubseteq^{i} D_{H}$ for each pair of combinatorial trees $G, H$.

In order to apply Theorem 4.2 we now define a reduction of $\cong^{i}$ on $\mathcal{D}$ to isomorphism on a suitable class of structures (the existence of such a reduction is a well-known result, see e.g. GK03, but here we need an explicit definition). Recall from [Kec95, Theorem 12.13] that there is a sequence $\psi_{n}: F(\mathbb{U}) \rightarrow \mathbb{U}$ of Borel functions such that for every $F \in F(\mathbb{U}) \backslash\{\emptyset\}$ the sequence $\left\langle\psi_{n}(F) \mid n \in \omega\right\rangle$ is an enumeration (which can be assumed without repetitions if $F$ is infinite) of a dense subset of $F$. If we consider the restriction of those $\psi_{n}$ to $\mathcal{D}$, the sequence defined 
above will actually be an enumeration of the full $F \in \mathcal{D}$, as each point of $F$ is isolated.

Consider the infinite language $\Lambda=\left\{R_{q} \mid q \in \mathbb{Q}^{+}\right\}$, where each $R_{q}$ is a binary predicate. To each $D=(X, d) \in \mathcal{D}$ associate the $\Lambda$-structure $S(D)$ on $\omega$ putting $R_{q}(i, j) \Longleftrightarrow d\left(\psi_{i}(X), \psi_{j}(X)\right)<q$. It is clear that if $D=(X, d), D^{\prime}=\left(X^{\prime}, d^{\prime}\right) \in \mathcal{D}$ and $\varphi$ is an isometry between $D$ and $D^{\prime}$, then the map $f_{\varphi}: \omega \rightarrow \omega$ which maps $i$ to the unique $j$ such that $\varphi\left(\psi_{i}(X)\right)=\psi_{j}\left(X^{\prime}\right)$ is an isomorphism between $S(D)$ and $S\left(D^{\prime}\right)$. Conversely, if $\rho$ is an isomorphism between $S(D)$ and $S\left(D^{\prime}\right)$, then the map $g_{\rho}: X \rightarrow X^{\prime}: \psi_{n}(X) \mapsto \psi_{\rho(n)}\left(X^{\prime}\right)$ is an isometry between $D$ and $D^{\prime}$.

Theorem 5.16. The relation of isometric embeddability between discrete Polish metric spaces is invariantly universal, meaning that for every analytic quasi-order $R$ there is a Borel class $\mathcal{C} \subseteq \mathcal{D}$ closed under isometry such that $R \sim_{B} \sqsubseteq^{i}\lceil\mathcal{C}$. In particular, $\sqsubseteq^{i}$ between arbitrary Polish metric spaces is invariantly universal.

Proof. Let $Z=\left\{F \in F(\mathbb{U}) \mid F\right.$ is infinite $\left.\wedge \forall n, m \in \omega d\left(\psi_{n}(F), \psi_{m}(F)\right) \in \omega\right\}$. So $Z$ is Borel and closed under isometry. Moreover, $Z \subseteq \mathcal{D}$ : indeed, for any $F \in Z$, notice that $F=\left\{\psi_{n}(F) \mid n \in \omega\right\}$ since $\left\{\psi_{n}(F) \mid n \in \omega\right\}$ is closed in $\mathbb{U}$, its only Cauchy sequences being the eventually constant ones; this same reason entails that $F$ is discrete.

It is enough now to show that the hypotheses of Theorem 4.2 are satisfied. As already observed above, the map which sends a combinatorial tree $G$ to the discrete Polish metric space $D_{G} \in Z$ simultaneously reduces $\sqsubseteq$ to $\sqsubseteq^{i}$ and $\cong$ to $\cong{ }^{i}$. Moreover the map which sends $D \in Z$ to the structure $S(D)$ is a reduction of $\cong i$ to $\cong$, and since $\cong$ is induced by the logic action of $S_{\infty}$ it remains to show that the map $\Sigma: \mathbb{G} \rightarrow G\left(S_{\infty}\right)$ which associates to each $G \in \mathbb{G}$ the group $H_{G}$ of the automorphisms of $S\left(D_{G}\right)$ is Borel. But it is easy to check that any non-trivial automorphism of $S\left(D_{G}\right)$ induces a non-trivial isometry of $D_{G}$ into itself, which in turn induces a non-trivial automorphism of $G$ : thus, since by Corollary 3.4 any $G \in \mathbb{G}$ is rigid, we get that each $H_{G}$ consists exactly of the identity function, therefore the map $G \mapsto H_{G}$ is constant (hence Borel).

We now consider the slightly more complicated case of ultrametric Polish spaces. In this case the collection $\mathcal{U}$ of all infinite ultrametric Polish spaces forms a standard Borel subspace of $F(\mathbb{U})$. We shall again apply Theorem 4.2, so we need a Borel reduction $f: \mathbb{G} \rightarrow \mathcal{U}$ of $\sqsubseteq$ into $\sqsubseteq^{i}$ and, simultaneously, of $\cong$ into $\cong^{i}$ and a Borel function $g$ reducing $\cong i$ on $\mathcal{U}$ to some orbit equivalence relation in a suitable way.

The function $f$ was (essentially) already defined in LR05 and FMR11: for $G \in \mathbb{G}$, let $U_{G}$ be the ultrametric Polish space consisting of all maximal paths of $G$ beginning in the root $\emptyset$ (such paths will be called branches of $G$ ), together with the metric $d_{G}$ defined by $d_{G}(x, y)=2^{-n}$, if branches $x$ and $y$ are distinct and share $n+1$ vertices of $G$, and $d_{G}(x, y)=0$ if $x=y$. Each $U_{G}$ is easily seen to be an ultrametric Polish space, and is isometrically identified in a Borel way with an element of $\mathcal{U}$, also denoted by $U_{G}$. Given any $G \in \mathbb{G}$, denote by $a_{x}, x \in{ }^{\omega} \omega$, the unique maximal branch which contains all vertices of the form $x \uparrow n$, by $b_{s, i}$ the branch determined by the point $\left(s^{++}, i, 0\right)$ (whenever such point exists in $G$ ), and by $c_{s, u, i}, u \in{ }^{<\omega} 2, i=0,1$, the branch determined by the point $\left(u, s, 0^{2 \theta(u)+2 \frown} i\right)$ (whenever this point exists in $G$ ). Let us call a fork any set of branches of the form $F_{s}^{G}=\left\{b_{s, i} \mid i \leq \# s+2\right\}$ or $F_{s, u}^{G}=\left\{c_{s, u, i} \mid i=0,1\right\}$ (where $s \in<\omega \omega, u \in<\omega^{<} 2$ ). Note that a point of $U_{G}$ is non-isolated if and only if it is of the form $a_{x}$. 
Lemma 5.17. Let $G, H \in \mathbb{G}$ and $U_{G}, U_{H}$ be defined as above. Then $U_{G} \cong{ }^{i} U_{H}$ implies $G=H$.

Proof. Note that any isometry $h$ between $U_{G}$ and $U_{H}$ must be a bijection of $\left\{a_{x} \mid x \in{ }^{\omega} \omega\right\}$ onto itself. It is sufficient to show that

Claim 5.17.1. Given $s \in{ }^{<\omega} \omega, u \in{ }^{<\omega} 2, F_{s, u}^{G}$ is a fork of $U_{G}$ if and only if $F_{s, u}^{H}$ is a fork of $U_{H}$.

To prove the claim we first show that $h\left(F_{s}^{G}\right)=F_{s}^{H}$. Note that all elements of $F_{s}^{G}$ are isolated, have distance $2^{-2|s|}$ from $a_{s^{\wedge} \overrightarrow{0}}$, have distances $2^{-(2|s|+2)}$ between them, and are the only points of $U_{G}$ with these properties. Let $t=y \uparrow|s|$ where $a_{y}=h\left(a_{s>\overrightarrow{0}}\right)$ : since $h$ is an isometry and the elements of $F_{t}^{H}$ are exactly the isolated points in $U_{H}$ with distance $2^{-2|s|}$ from $a_{y}$ and distance $2^{-(2|s|+2)}$ between them, we conclude that $h\left(F_{s}^{G}\right)=F_{t}^{H}$. In particular, $F_{s}^{G}$ and $F_{t}^{H}$ have the same cardinality, hence $s=t$.

This also implies that $h\left(a_{x}\right)=a_{x}$ for every $x \in{ }^{\omega} \omega$. Now note that the elements of $F_{s, u}^{G}$ are exactly the two points of $U_{G}$ which have distance $2^{-2|s|}$ from $a_{s\urcorner \overrightarrow{0}}$, and have distance $2^{-(2|s|+2 \theta(u)+3)}$ between them. But then $h$ maps them to two points of $U_{H}$ which have distance $2^{-2|s|}$ from $a_{s\urcorner \overrightarrow{0}}$, and have distance $2^{-(2|s|+2 \theta(u)+3)}$ between them. Such points can only be the elements of $F_{s, u}^{H}$, which is therefore a fork of $U_{H}$.

Lemma 5.18. Let $G, H \in \mathbb{G}$. Then $G \sqsubseteq H \Longleftrightarrow U_{G} \sqsubseteq^{i} U_{H}$.

Proof. We just show $U_{G} \sqsubseteq^{i} U_{H} \Rightarrow G \sqsubseteq H$ because any embedding between $G$ and $H$ can be easily converted into an isometric embedding between $U_{G}$ and $U_{H}$.

Fix an isometric embedding $h$ from $U_{G}$ to $U_{H}$. Note that $h$ must send elements of the form $a_{x}$ into elements of the same form. The function $h$ induces the Lipschitz $\operatorname{map} \varphi:<\omega \omega \rightarrow{ }^{<\omega} \omega$ defined by $\varphi(s)=y\left\lceil|s|\right.$ where $a_{y}=h\left(a_{s>\overrightarrow{0}}\right)$.

We first show that if $s \in<\omega \omega, u \in{ }^{<\omega} 2$ are such that $F_{s, u}^{G}$ is a fork of $U_{G}$, then $F_{\varphi(s), u}^{H}$ is a fork of $U_{H}$. In fact, as already noticed in the proof of the previous lemma, the elements of $F_{s, u}^{G}$ are exactly the two points of $U_{G}$ which have distance $2^{-2|s|}$ from $a_{s \rightarrow \overrightarrow{0}}$, and have distance $2^{-(2|s|+2 \theta(u)+3)}$ between them. But then there must be two points in $U_{H}$ which have distance $2^{-2|s|}$ from $a_{y}$ (where $y$ is as in the definition of $\varphi$ ), and have distance $2^{-(2|s|+2 \theta(u)+3)}$ between them: this can happen only if $F_{\varphi(s), u}^{H}$ is a fork of $U_{H}$.

Now let $S$ and $T$ be normal trees such that $G=G_{S}$ and $H=G_{T}$. By the above, $\varphi$ is a witness of $S \leq_{\max } T$, and hence $G \sqsubseteq H$.

Regarding the map $g$ that will be used in Theorem 5.19, it was already introduced in [GK03, although here we need to perform a slight modification to the original construction in order to simplify subsequent computations. Let $\psi_{n}: F(\mathbb{U}) \rightarrow \mathbb{U}$ be Borel functions defined as above. The basic open balls of a space $U \in \mathcal{U}$ are of the form $B\left(\psi_{n}(U), q\right)=\left\{x \in U \mid d_{U}\left(x, \psi_{n}(U)\right)<q\right\}$ for some $n \in \omega$ and $q \in \mathbb{Q}^{+}$. Let $\Lambda$ be a first order language consisting of a binary relation symbol $R$ and of countably many unary relation symbols $Q_{r}$, for $r \in \mathbb{Q}^{+}$. For each $U \in \mathcal{U}$, consider the $\Lambda$-structure $S(U)$ whose domain is $\left\{(n, q) \mid n \in \omega, q \in \mathbb{Q}^{+}\right\}$, in which $R$ holds between $(n, q)$ and $\left(n^{\prime}, q^{\prime}\right)$ if and only if $B\left(\psi_{n}(U), q\right) \subseteq B\left(\psi_{n^{\prime}}(U), q^{\prime}\right)$, and in which $Q_{r}$ holds for $(n, q)$ if and only if $\operatorname{diam}\left(B\left(\psi_{n}(U), q\right)\right)<r$. As it is shown in GK03, 
the map sending $U \in \mathcal{U}$ to $S(U)$ (coded as a $\Lambda$-structure with domain $\omega$ ) is Borel and reduces $\cong{ }^{i}$ to $\cong$.

Notice now that the above construction does not really depend on the choice of the functions $\psi_{n}$. Had we chosen a different sequence of functions $\psi_{n}^{\prime}$ (with the same properties), the structure constructed from $U \in \mathcal{U}$ as explained above but using the $\psi_{n}^{\prime}$ instead of the $\psi_{n}$ would be formally different but still isomorphic to $S(U)$. So define the $\psi_{n}^{\prime}$ as follows: first set $\psi_{n}^{\prime} \uparrow(F(\mathbb{U}) \backslash f(\mathbb{G}))=\psi_{n} \uparrow(F(\mathbb{U}) \backslash f(\mathbb{G}))$. Then, for $G \in \mathbb{G}$

- let $\psi_{3 \# s}^{\prime}\left(U_{G}\right)=a_{s \frown \overrightarrow{0}}$,

- let $\left\langle\psi_{3 n+1}^{\prime}\left(U_{G}\right) \mid n \in \omega\right\rangle$ be the enumeration of the branches of $G$ of the form $b_{s, i}$ according to the position of $(s, i)$ in some fixed ordering of $<\omega \omega \times \omega$ of type $\omega$,

- let $\left\langle\psi_{3 n+2}^{\prime}\left(U_{G}\right) \mid n \in \omega\right\rangle$ be the enumeration of the branches of $G$ of the form $c_{s, u, i}$ according to the position of $(s, u, i)$ in some fixed ordering of ${ }^{<\omega} \omega \times{ }^{<\omega} 2 \times 2$ of type $\omega$.

Since $f(\mathbb{G})$ is a Borel subset of $\mathcal{U}$ (as $\mathbb{G}$ is Borel and $f$ is injective because it reduces equality on $\mathbb{G}$ to isometry), the functions $\psi_{n}^{\prime}$ are still Borel and their values on $U \in \mathcal{U}$ still enumerate a dense subset of $U$. Still call $S(U)$ the $\Lambda$-structure constructed from $U \in \mathcal{U}$ as explained above but using these specific $\psi_{n}^{\prime}$ in the construction (each $S(U)$ will be confused with its Borel-in- $U$ coded version as a $\Lambda$-structure on $\omega$ ), and let $g$ be the Borel map sending $U$ to $S(U)$.

Theorem 5.19. The relation of isometric embeddability between ultrametric Polish spaces is invariantly universal, that is, for every analytic quasi-order $R$ there is a Borel class $\mathcal{C} \subseteq \mathcal{U}$ closed under isometry such that $R \sim_{B} \sqsubseteq^{i} \uparrow \mathcal{C}$.

Proof. To show that the hypotheses of Theorem 4.2 are satisfied, consider the maps $f$ and $g$ defined above: by Lemmas 5.17 and 5.18 and the previous observations, we just need to check that the map $\Sigma$ sending $G \in \mathbb{G}$ into the group of automorphisms of $S\left(U_{G}\right)$ is Borel.

First note that, by the proof of Lemma [5.17, an isometry of $U_{G}$ into itself must be the identity on branches of the form $a_{x}$ (i.e. on its accumulation points) and set-wise fix the forks of $U_{G}$. Then notice that the function taking $G$ into the binary relation $D_{G}$ on $\omega$, defined by

$$
\begin{aligned}
n D_{G} m \Longleftrightarrow \quad \psi_{n}^{\prime}\left(U_{G}\right) \text { and } \psi_{m}^{\prime}\left(U_{G}\right) \text { are both isolated } \\
\text { and belong to the same fork of } G,
\end{aligned}
$$

is Borel. Finally, recall from GK03 that any automorphism $H$ of $S\left(U_{G}\right)$ induces the isometry $H^{\prime}: U_{G} \rightarrow U_{G}$ defined by $H^{\prime}(x)=y \Longleftrightarrow\{y\}=\bigcap_{k \in \omega} B\left(\psi_{m_{k}}\left(U_{G}\right), q_{k}\right)$, where the $m_{k}$ and the $q_{k}$ are such that there are sequences $\left\langle n_{k} \mid k \in \omega\right\rangle,\left\langle r_{k} \mid k \in \omega\right\rangle$ of natural numbers and elements of $\mathbb{Q}^{+}$, respectively, with the properties that $\psi_{n_{k}}\left(U_{G}\right) \rightarrow x, r_{k} \rightarrow 0$, and $H\left(n_{k}, r_{k}\right)=\left(m_{k}, q_{k}\right)$ for every $k \in \omega$. From all these facts it follows that given a function $h: A \subseteq \omega \times \mathbb{Q}^{+} \rightarrow \omega \times \mathbb{Q}^{+}$with $A$ finite, the set of those $G \in \mathbb{G}$ for which $h$ can be extended to an automorphism of $S\left(U_{G}\right)$ is defined by the conjunction of the following Borel conditions:

- $h$ is a partial automorphism of $S\left(U_{G}\right)$ (i.e. it is injective and respects the predicates $R, Q_{r}$ of $S\left(U_{G}\right)$ );

- for every $(n, q) \in A$, if there is $r \in \mathbb{Q}^{+}$such that $Q_{r}$ does not hold for $(n, q)$ in $S\left(U_{G}\right)$, then $(n, q) R h(n, q) R(n, q)$ (in $S\left(U_{G}\right)$ ); 
- for every $(n, q) \in A$, if $Q_{r}$ holds for $(n, q)$ in $S\left(U_{G}\right)$ for every $r \in \mathbb{Q}^{+}$and $h(n, q)=\left(n^{\prime}, q^{\prime}\right)$, then $n D_{G} n^{\prime}$.

Indeed, fix $G \in \mathbb{G}$ and for $(n, q) \in \omega \times \mathbb{Q}^{+}$put $B_{n, q}=B\left(\psi_{n}\left(U_{G}\right), q\right)$. Now notice that for each $(n, q) \in \omega \times \mathbb{Q}^{+}$there are infinitely many distinct pairs $\left(n^{\prime}, q^{\prime}\right) \in \omega \times \mathbb{Q}^{+}$ such that $B_{n, q}=B_{n^{\prime}, q^{\prime}}$ (so that in $S\left(U_{G}\right)$ we have $(n, q) R\left(n^{\prime}, q^{\prime}\right) R(n, q)$ and $Q_{r}(n, q) \Longleftrightarrow Q_{r}\left(n^{\prime}, q^{\prime}\right)$ for every $\left.r \in \mathbb{Q}^{+}\right)$: such pairs will be called names for the basic ball $B_{n, q}$. If $h$ satisfies all the conditions above, then it can be extended to an automorphism $H$ of $S\left(U_{G}\right)$ in the following way. Let $(n, q) \in A$ and let $(m, r)=h(n, q)$ : if $B_{n, q}$ contains two distinct points, then $B_{n, q}=B_{m, r}$, while if $B_{n, q}$ isolates some point $x \in U_{G}$, then $B_{m, r}$ isolates a point which belongs to the same fork of $x$ (hence $h$ induces partial permutations on the forks of $U_{G}$ ). Define $H$ on the names of $B_{n, q}$ by choosing any bijection extending $h$ between such names and the names of $B_{m, r}$ (this can be done because $A$ is finite and every basic ball has infinitely many names). For any other pair $\left(n^{\prime}, q^{\prime}\right)$ not yet considered (that is for any element of $\omega \times \mathbb{Q}^{+}$which is not the name of $B_{n, q}$ for some $\left.(n, q) \in A\right)$, we consider two cases: if $B_{n^{\prime}, q^{\prime}}$ does not isolate a point of $U_{G}$, define $H\left(n^{\prime}, q^{\prime}\right)=\left(n^{\prime}, q^{\prime}\right)$. Otherwise, $B_{n^{\prime}, q^{\prime}}=\{x\}$ where $x$ belongs to some fork $F$ of $U_{G}$. Choose any permutation of the elements of $F$ which extends the partial permutation induced by $h$ : if $x$ is sent to some $y$ by such permutation, then define $H$ on the names of $B_{n^{\prime}, q^{\prime}}$ by choosing any permutation between such names and the names of the basic ball which isolates $y$. It is not hard to check that the $H$ constructed in this way is an automorphism of $S\left(U_{G}\right)$. Conversely, if $H$ is an automorphism of $S\left(U_{G}\right)$ such that $h=H \uparrow A$, then the whole $H$ must satisfy the conditions above because, as observed above, the isometry $H^{\prime}$ of $U_{G}$ into itself induced by $H$ must be the identity on its accumulation points and set-wise fix all its forks; therefore, $h$ satisfies such conditions as well.

5.6. Linear isometric embeddings between separable Banach spaces. Any separable Banach space $X=\left(X,\|\cdot\|_{X}\right)$ is linearly isometric to a closed subspace of $\mathcal{C}([0,1])$ equipped with the sup norm, hence it is natural to consider the Borel set $\mathcal{B}$ of all closed linear subspaces of $\mathcal{C}([0,1])$ as the standard Borel space of separable Banach spaces. For $X, Y \in \mathcal{B}$ say that $X$ linearly isometrically embeds into $Y$ ( $X \sqsubseteq^{\text {li }} Y$ in symbols) if there is a linear isometric embedding, that is a linear and norm-preserving map, between $X$ and $Y$. The relation $\sqsubseteq^{\text {li }}$ is obviously an analytic quasi-order, and the relation $\cong{ }^{\mathrm{li}}$ of linear isometry is the corresponding

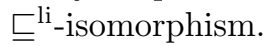

In [LR05] it was proved that $\check{c}^{\text {li }}$ is a complete analytic quasi-order, so we would like to improve this result by showing that $\complement^{\text {li }}$ is indeed invariantly universal (coupled with $\cong$ li $)$. In order to apply Theorem 4.2, we need a Borel map $f$ simultaneously reducing embeddability and isomorphism on $\mathbb{G}$ to, respectively, $\coprod^{\mathrm{li}}$ and $\stackrel{\mathrm{li}}{ }^{\mathrm{i}}$, and then a Borel map $g$ reducing $\cong$ li (possibly restricted to some standard Borel space $Z$ invariant under linear isometry with $f(\mathbb{G}) \subseteq Z \subseteq \mathcal{B}$, so that ${ }^{\text {li }}$-saturation in $Z$ will coincide with $\cong$ li -saturation in $\mathcal{B}$ ) to some orbit equivalence relation in such a way that the map $\Sigma$ assigning to each $G \in \mathbb{G}$ the stabilizer of $g(f(G))$ is Borel. A map $f$ with the required properties was already defined in LR05, Theorem 4.6]. Moreover, $\cong{ }^{\mathrm{li}}$ (on the whole $\mathcal{B}$ ) is reducible to an orbit equivalence relation: this is because by a theorem of Mazur $\cong \mathrm{l}$ and $\cong \mathrm{i}$ coincide on $\mathcal{B}$, and by GK03. the relation $\cong \mathrm{i}$ is in turn Borel reducible to (in fact, Borel bireducible with) the orbit equivalence relation on $F(\mathbb{U})$ induced by the natural action of the group of 
automorphisms of the Urysohn space $\mathbb{U}$. However, the last reduction is not quite explicit, and hence it seems to be very difficult to have a control on the resulting map $\Sigma$. Therefore we will restrict our attention to a suitable proper $\cong$ li -saturated $Z \subseteq \mathcal{B}$, and to define such $Z$ we will first need to slightly modify the original construction of the map $f$ from LR05.

Let $c_{0}$ be the Banach space of sequences converging to 0 endowed with the supnorm $\|\cdot\|_{\infty}$, let $\left\langle e_{p} \mid p \in \omega\right\rangle$ be the usual base of $c_{0}$, and denote elements of $c_{0}$ by $\Sigma_{n} \alpha_{n} e_{n}$. Given $G \in \mathbb{G}$ define 3 a new norm $\|\cdot\|_{G}$ on $c_{0}$ by

$$
\left\|\Sigma_{n} \alpha_{n} e_{n}\right\|_{G}=\sup \left\{\left|\alpha_{i}\right|+\frac{\left|\alpha_{j}\right|}{3-\chi_{G}(i, j)} \mid i \neq j \in \omega\right\}
$$

where $\chi_{G}: \omega \times \omega \rightarrow\{0,1\}$ is the characteristic function of the graph relation of $G$. As for the norms defined in the proof of [LR05. Theorem 4.6], it is easy to check that $\|\cdot\|_{G}$ is indeed equivalent to $\|\cdot\|_{\infty}$, as $\left\|\Sigma_{n} \alpha_{n} e_{n}\right\|_{\infty} \leq\left\|\Sigma_{n} \alpha_{n} e_{n}\right\|_{G} \leq \frac{3}{2}\left\|\Sigma_{n} \alpha_{n} e_{n}\right\|_{\infty}$, and that the map $f$ sending $G \in \mathbb{G}$ into $X_{G}=\left(c_{0},\|\cdot\|_{G}\right)$ is Borel (when we identify $\left(c_{0},\|\cdot\|_{G}\right)$ with its linearly isometric copy in $\left.\mathcal{B}\right)$.

Lemma 5.20. The map $f$ reduces $\sqsubseteq$ to $\sqsubseteq^{\mathrm{li}}$ and $\cong t_{o} \cong$ li .

Proof. The proof is almost identical to that of [LR05, Theorem 4.6]. If $h$ is an embedding of $G$ into $H$, then $\Sigma_{n} \alpha_{n} e_{n} \mapsto \Sigma_{n} \alpha_{n} e_{h(n)}$ is a linear isometric embedding of $X_{G}$ into $X_{H}$.

Conversely, for any linear isometric embedding $h^{\prime}: X_{G} \rightarrow X_{H}$, one first proves (copying the original proof word by word) that there is $h: \omega \rightarrow \omega$ such that for every $p \in \omega$ there is $\epsilon_{p} \in\{1,-1\}$ for which $h^{\prime}\left(e_{p}\right)=\epsilon_{p} e_{h(p)}$. Arguing as in the original proof one gets that for $p \neq q$ it is true that $h(p) \neq h(q)$ and moreover

$$
\begin{aligned}
1+\frac{1}{3-\chi_{G}(p, q)}=\left\|\epsilon_{p} e_{p}+\epsilon_{q} e_{q}\right\|_{G} & =\left\|h^{\prime}\left(\epsilon_{p} e_{p}+\epsilon_{q} e_{q}\right)\right\|_{H} \\
& =\left\|e_{h(p)}+e_{h(q)}\right\|_{H}=1+\frac{1}{3-\chi_{H}(h(p), h(q))},
\end{aligned}
$$

so that $h$ is an embedding between $G$ and $H$ because $\chi_{G}(p, q)=\chi_{H}(h(p), h(q))$.

As noted in [LR05, Remark 4.7], the above construction also yields the result about isomorphism and linear isometry.

It is not hard to check that in a Banach space of the form $X_{G}$ the elements $e_{p}$ of the base and their opposite $-e_{p}$ are the unique extreme points of the unit ball. Consider the set

$$
E=\{(x, X) \in \mathcal{C}([0,1]) \times \mathcal{B} \mid x \text { is an extreme point of the unit ball of } X\} .
$$

It is straightforward to check that $E$ is co-analytic, and it follows from a result of Kaufman ( Kau00, Section III]) that $E$ is not Borel. This means that we cannot use $E$ to make our subsequent computations work. Therefore we use the following stronger notion.

\footnotetext{
${ }^{3}$ The norm $\|\cdot\|_{G}$ (and hence the Banach space $X_{G}$ ) can actually be defined starting from any graph $G$, and not just from graphs in $\mathbb{G}$. Moreover, Lemma 5.20 would still hold in this more general setup.
} 
Definition 5.21. A point $x$ of the unit ball $B_{X}$ of the Banach space $X$ is strongly extreme if and only if

$$
\forall \varepsilon>0 \exists \delta>0 \forall y, z \in B_{X}\left(\left\|x-\frac{y+z}{2}\right\|_{X} \leq \delta \Rightarrow\|y-z\|_{X} \leq \varepsilon\right) .
$$

Obviously, if $x \in X$ is a strongly extreme point of $B_{X}$, then it is also an extreme point of $B_{X}$ (but the converse does not hold in general). Moreover, it is easy to check using standard arguments that

$S E=\{(x, X) \in \mathcal{C}([0,1]) \times \mathcal{B} \mid x$ is a strongly extreme point of the unit ball of $X\}$

is Borel. In fact it is enough to check that $(x, X) \in S E$ if and only if it satisfies the restricted version of (1) obtained by considering just $\varepsilon, \delta \in \mathbb{Q}$ and $y, z$ ranging over some countable dense subset of $B_{X}$.

Lemma 5.22. For any $G \in \mathbb{G}, \epsilon \in\{-1,1\}$ and $p \in \omega, \epsilon e_{p}$ is a strongly extreme point of $B_{X_{G}}$.

Proof. Given $\varepsilon>0$ put $\delta=\frac{1}{18} \varepsilon$. Let $y=\Sigma_{n} \beta_{n} e_{n}, z=\Sigma_{n} \gamma_{n} e_{n} \in B_{X_{G}}$ be such that $\left\|e_{p}-\frac{y+z}{2}\right\|_{G} \leq \delta$. The hypotheses on $y, z$ easily imply $1-2 \delta \leq\left|\beta_{p}\right|,\left|\gamma_{p}\right| \leq 1$. But then for $n \neq p$ we must have $\left|\beta_{n}\right|,\left|\gamma_{n}\right| \leq 6 \delta$ since $y$ and $z$ both belong to the unit ball, whence $\left|\beta_{n}-\gamma_{n}\right| \leq 12 \delta$ for every $n \in \omega$. This implies that for every $i \neq j \in \omega$

$$
\left|\beta_{i}-\gamma_{i}\right|+\frac{\left|\beta_{j}-\gamma_{j}\right|}{3-\chi_{G}(i, j)} \leq 12 \delta+\frac{12 \delta}{2}=18 \delta=\varepsilon,
$$

as required.

Since all the points of $X_{G}$ which are not of the form $\epsilon e_{p}$ are not even extreme points of $B_{X_{G}}$, from the previous lemma we can conclude that $\left\{\epsilon e_{p} \mid \epsilon \in\{-1,1\}\right.$, $p \in \omega\}$ is the collection of all strongly extreme points of $B_{X_{G}}$.

Notice that the definition of $X_{G}$ in LR05 is such that the unit ball has no strongly extreme points. This is the reason why we had to change such a definition.

Now let $A$ be the closure under linear isometry of $f(\mathbb{G})$ and

$$
B=\left\{X \in \mathcal{B} \mid \forall x, y\left(S E(x, X) \wedge S E(y, X) \Rightarrow\|x-y\|_{X}>1\right)\right\} .
$$

Both $A, B$ are invariant with respect to $\cong$ li,$A$ is analytic, $B$ is co-analytic, and $A \subseteq B$ by direct computation of the values of $\left\|e_{i}-e_{j}\right\|_{G}$ for $i \neq j \in \omega, G \in \mathbb{G}$. Therefore by [Kec95, Exercise 14.14] there is a Borel set $C$ which is $\cong$ li -invariant and such that $A \subseteq C \subseteq B$. Notice that for each element of $C$ there are at most countably many strongly extreme points of its unit ball, as the same property clearly holds for all elements of $B$ by separability of the Banach spaces under consideration. This means that the Borel set

$$
D=\{(X, x) \in C \times \mathcal{C}([0,1]) \mid S E(x, X)\}
$$

has countable vertical sections, so that by [Kec95, Exercise 18.15],

$$
C_{\omega}=\left\{X \in C \mid B_{X} \text { has } \omega \text {-many strongly extreme points }\right\}
$$

is Borel as well, and there is a sequence $f_{n}: C_{\omega} \rightarrow \mathcal{C}([0,1])$ of Borel functions such that $\left\langle f_{n}(X) \mid n \in \omega\right\rangle$ is an enumeration without repetitions of the strongly extreme points of $B_{X}$ (for every $X \in C_{\omega}$ ). Notice also that $C_{\omega}$ is obviously $\cong$ ii-invariant and contains $f(\mathbb{G})$.

Now we further refine the set $C_{\omega} \subseteq \mathcal{B}$. Let $\psi_{n}: C_{\omega} \rightarrow \mathcal{C}([0,1])$ be a sequence of Borel functions such that for every $X \in C_{\omega}$ the set $\left\{\psi_{n}(X) \mid n \in \omega\right\}$ is a dense 
subset of $X$. Given $X \in C_{\omega}$, put $X \in Z$ if and only if for every $\varepsilon \in \mathbb{Q}^{+}$and every $m \in \omega$ there are $n \in \omega, \alpha_{0}, \ldots, \alpha_{n} \in \mathbb{Q} \backslash\{0\}$, and $k_{0}, \ldots, k_{n} \in \omega$ such that $\left\|\psi_{m}(X)-\left(\alpha_{0} f_{k_{0}}(X)+\cdots+\alpha_{n} f_{k_{n}}(X)\right)\right\|_{X} \leq \varepsilon$, that is, if and only if the rational linear combinations of strongly extreme points of $B_{X}$ are dense in $X$.

It is easy to check that $Z$ is Borel, $\cong$ li -invariant, and contains $f(\mathbb{G})$. The motivation in restricting our attention to this particular $Z$ is that, as we will see, $\cong$ li on $Z$ is actually Borel reducible to a relation of isomorphism on countable structures. Indeed, let $\Lambda$ be the language containing all $(n+1)$-ary relational symbols of the form $R_{q}^{\alpha_{0}, \ldots, \alpha_{n}}$ for $n \in \omega, \alpha_{0}, \ldots, \alpha_{n} \in \mathbb{Q}$, and $q \in \mathbb{Q}^{+}$. Given $X \in Z$, define the $\Lambda$-structure $S(X)$ on $\omega$ by stipulating that for each $k_{0}, \ldots, k_{n} \in \omega$ the predicate $R_{q}^{\alpha_{0}, \ldots, \alpha_{n}}\left(k_{0}, \ldots, k_{n}\right)$ holds in $S(X)$ if and only if $\left\|\alpha_{0} f_{k_{0}}(X)+\cdots+\alpha_{n} f_{k_{n}}(X)\right\|_{X}<q$.

Lemma 5.23. The function $g: Z \rightarrow \operatorname{Mod}_{\Lambda}: X \mapsto S(X)$ is a Borel reduction of $\cong$ li to $\cong$.

Proof. Clearly $g$ is a Borel map. Let $X, Y \in Z$ be such that $X \cong{ }^{\text {li }} Y$ via some $h$ : then $h$ must be a bijection between the strongly extreme points of $B_{X}$ and $B_{Y}$, and therefore it induces in the obvious way a permutation $H$ of $\omega$ such that $h\left(f_{n}(X)\right)=$ $f_{H(n)}(Y)$ for every $n \in \omega$. Moreover, since $h$ is linear and norm-preserving it is easy to verify that for every $\alpha_{0}, \ldots, \alpha_{n} \in \mathbb{Q}, q \in \mathbb{Q}^{+}$and $k_{0}, \ldots, k_{n} \in \omega$, one has that $R_{q}^{\alpha_{0}, \ldots, \alpha_{n}}\left(k_{0}, \ldots, k_{n}\right)$ holds in $S(X)$ if and only if $R_{q}^{\alpha_{0}, \ldots, \alpha_{n}}\left(H\left(k_{0}\right), \ldots, H\left(k_{n}\right)\right)$ holds in $S(Y)$, so that $S(X) \cong S(Y)$.

Conversely, let $H$ be an isomorphism between $S(X)$ and $S(Y)$, and consider the linear extension $h$ of the map $f_{n}(X) \mapsto f_{H(n)}(Y)$ to the sets of vectors which are rational linear combinations of the extreme points of $B_{X}$ and $B_{Y}$ (denoted as $R L C_{X}$ and $R L C_{Y}$, respectively). To show that $h$ is well defined, note first that it is norm-preserving by the definition of the predicates $R_{q}^{\alpha_{0}, \ldots, \alpha_{n}}$ in $S(X)$ and $S(Y)$. In particular, $h$ is well defined on the zero vector, and its image is the zero vector. Let $\alpha_{0}, \ldots, \alpha_{n}, \beta_{0}, \ldots, \beta_{m} \in \mathbb{Q}, k_{0}, \ldots, k_{n}, l_{0}, \ldots, l_{m} \in \omega$ be such that $\sum_{i=0}^{n} \alpha_{i} f_{k_{i}}(X)=$ $\sum_{j=0}^{m} \beta_{0} f_{l_{j}}(X)$. Then $\sum_{i=0}^{n} \alpha_{i} f_{H\left(k_{i}\right)}(Y)-\sum_{j=0}^{m} \beta_{j} f_{H\left(l_{j}\right)}(Y)$ is the image under $h$ of $\sum_{i=0}^{n} \alpha_{i} f_{k_{i}}(X)-\sum_{j=0}^{m} \beta_{0} f_{l_{j}}(X)=0$. So $\sum_{i=0}^{n} \alpha_{i} f_{H\left(k_{i}\right)}(Y)-\sum_{j=0}^{m} \beta_{j} f_{H\left(l_{j}\right)}(Y)=0$. Similarly, one can prove that $h$ is injective. Being surjective too, $h$ is a bijection $R C L_{X} \rightarrow R C L_{Y}$.

By definition, $h$ is linear with respect to rational linear combinations, i.e. if $v, w \in R L C_{X}$ and $\alpha, \beta \in \mathbb{Q}$, then $h(\alpha v+\beta w)=\alpha h(v)+\beta h(w)$. But since $R L C_{X}$ and $R L C_{Y}$ are dense in $X$ and $Y$ (respectively) by the definition of $Z$, using standard arguments we have that $h$ extends to a (unique) linear isometry between $X$ and $Y$, so that $X \cong$ li $Y$.

Now we are ready to prove that $\sqsubseteq^{\text {li }}$ is invariantly universal.

Theorem 5.24. The relation of linear isometric embeddability $\sqsubseteq^{\text {li }}$ between separable Banach spaces is invariantly universal, that is, for every analytic quasi-order $R$, there is a Borel class $\mathcal{C} \subseteq \mathcal{B}$ closed under linear isometry such that $R \sim_{B} \sqsubseteq^{\mathrm{li}} \uparrow \mathcal{C}$.

Proof. We want to apply Theorem 4.2 to the maps $f$ and $g$ previously defined. Having already proved in Lemmas 5.20 and 5.23 that $f$ is as desired and $g$ reduces the linear isometry relation on $Z$ to the isomorphism relation, it remains only to show that the map $\Sigma$ assigning to each $G \in \mathbb{G}$ the group of automorphisms of $S\left(X_{G}\right)$ is Borel. First notice that the map sending $G \in \mathbb{G}$ into the binary relation $O_{G}$ on $\omega$ defined by $n O_{G} m \Longleftrightarrow f_{n}\left(X_{G}\right)=-f_{m}\left(X_{G}\right)$ is Borel. Given an injective sequence 
$s \in{ }^{<\omega} \omega$, we now have that $S\left(X_{G}\right)$ has an automorphism extending $s$ if and only if for every $i<|s|$, either $s_{i}=i$ or $s_{i} O_{G} i$ : in fact, the proofs of Lemmas 5.20 and 5.23 show that any automorphism $H$ of $S\left(X_{G}\right)$ such that $H(n) \neq n$ and $\neg\left(H(n) O_{G} n\right)$ would induce a linear isometry $h$ of $X_{G}$ into itself such that $h\left(e_{n}\right)=\epsilon e_{H(n)}$ for $\epsilon \in\{1,-1\}$, which in turn would naturally induce a non-trivial automorphism of $G$ sending $n$ into $H(n)$, contradicting Corollary 3.4. Therefore, $\Sigma$ is Borel, and we are done.

5.7. Further applications. In this section we will consider the remaining complete analytic quasi-orders which have appeared in the literature, and sketch the proofs that they are indeed invariantly universal.

Following Cam05a, given two subcontinua $K, K^{\prime}$ of a Hilbert cube, we put

- $K \preceq K^{\prime}$ if there is a continuous surjection $K^{\prime} \rightarrow K$;

- $K \preceq_{M} K^{\prime}$ if there is a monotone surjection $K^{\prime} \rightarrow K$;

- $K \preceq_{R} K^{\prime}$ if there is an $r$-mapping $K^{\prime} \rightarrow K$;

- $K \preceq_{W} K^{\prime}$ if there is a weakly confluent surjection $K^{\prime} \rightarrow K$;

- $K \preceq_{O} K^{\prime}$ if there is an open continuous surjection $K^{\prime} \rightarrow K$.

Clearly the isomorphism associated to each of these morphism relations is always the relation of homeomorphism $\simeq$. We start by considering the quasi-order $\preceq$ and give some hints on how to prove that the pair $(\preceq, \simeq)$ is indeed invariantly universal (we leave the details to the reader). In [Cam05a, Theorem 3] a Borel map $f$ was provided from countable graphs into compacta which reduces the embeddability relation to $\preceq$. As done in subsection [5.1] it is not hard to check (using a back and forth argument for the non-trivial direction) that $f$ simultaneously reduces the isomorphism relation to $\simeq$.

In order to apply Theorem 4.2 we now would need to reduce $\simeq$ to some orbit equivalence relation. Notice that, as observed e.g. in [LR05, Section 4.2], $\simeq$ is reducible to the natural action of the automorphism group of the Urysohn space $\mathbb{U}$ on its closed subspaces (as by Banach-Stone $K \simeq K^{\prime} \Longleftrightarrow\left(\mathcal{C}(K),\|\cdot\|_{\infty}\right) \cong$ li $\left.\left(\mathcal{C}\left(K^{\prime}\right),\|\cdot\|_{\infty}\right)\right)$. Unfortunately, with this reduction we lack control on the complexity of the map assigning to each compacta the stabilizer of its image under this reduction. Nevertheless, we can use an approach similar to the one of subsection 5.6. i.e. we can restrict our attention to a suitable Borel class $Z$ of compacta closed under homeomorphism and containing $f(\mathbb{G})$, and provide an ad hoc reduction of $\simeq\lceil Z$ to the isomorphism relation which satisfies the hypotheses of Theorem 4.2 .

However, in this particular case we can also use an even simpler argument to prove the invariant universality of $\preceq$ : in fact, by the properties of Cook (sub)continua, the rigidity of each $G \in \mathbb{G}$ and the definition of the map $f$, we have that for every Borel $B \subseteq \mathbb{G}$, a compactum $K$ belongs to the $\simeq$-saturation of $f(B)$ if and only if there is a unique pair $(G, h)$ such that $G \in B$ and $h$ is a homeomorphism between $f(G)$ and $K$ (for more details we refer the reader to Cam05a). Therefore the $\cong$-saturation of $f(B)$ is a Borel set by the Luzin theorem Kec95, Theorem 18.11] again, and as shown in the proof of Theorem 4.2 this fact implies that $(\preceq, \simeq)$ is invariantly universal.

Notice that this result automatically extends to the quasi-orders $\preceq_{M}, \preceq_{R}$ and $\preceq_{W}$ (see the explanation in Cam05a, p. 203]), and even to any quasi-order $S$ on compacta such that $\preceq_{M} \cap \preceq_{R} \subseteq S \subseteq \preceq$ (when paired with $\simeq$ ). Moreover, it is not hard to show (applying Theorem 4.2 again) that $\preceq_{O}$ too is invariantly 
universal. This is because in Cam05a it was proved that embeddability between graphs is reducible to $\preceq_{O}$ restricted to (very simple) dendrites, so that it is enough to combine the techniques developed in subsections 5.1 and 5.4 to get the desired result. Therefore we have the following:

Theorem 5.25. The quasi-orders $\preceq_{,} \preceq_{M}, \preceq_{R}, \preceq_{W}, \preceq_{O}$ are all invariantly universal. Moreover, for every quasi-order $S$ such that $\preceq_{M} \cap \preceq_{R} \subseteq S \subseteq \preceq$, the pair $(S, \simeq)$ is invariantly universal.

\section{Open PROBlems}

We conclude the paper by stating a few questions about invariantly universal analytic pairs which, in our opinion, deserve further investigation.

Question 6.1. Is the pair $(S,=)$ invariantly universal whenever $S$ is a complete analytic quasi-order?

Notice that if $S$ witnesses that the answer to the previous question is negative, then $(S, E)$ is not invariantly universal for all analytic equivalence relations $E$ (since $=\subseteq E)$.

A more general question is the following:

Question 6.2. Suppose that $(S, F)$ is invariantly universal, $E \subseteq E_{S}$ and $E \leq_{B} F$. Does it follow that $(S, E)$ is also invariantly universal?

The intuition behind Question 6.2 is that when $E \leq_{B} F$ it should be easier to find $E$-invariant sets than to find $F$-invariant sets.

The last questions (which are related) are necessarily less precise, as they involve the notions of natural morphism relation and natural pair. Here a natural morphism relation is any morphism relation which is of independent interest in some area of mathematics, while a natural pair would typically consist of a quasi-order which is a natural morphism relation and of its isomorphism relation (where both the morphism relation and its isomorphism relation are intended to be analytic).

Question 6.3. Is there a natural pair $(S, E)$ which is not invariantly universal, although $S$ is a complete analytic quasi-order?

Recall that in the introduction we gave an example of analytic quasi-orders $S$ and $R$ such that $\left(S, E_{S}\right)$ is invariantly universal while $\left(R, E_{R}\right)$ is not. The next question asks whether there exist (natural) morphism relations behaving in each of these ways.

Question 6.4. Is there a natural morphism relation $S$ such that $\left(S, E_{S}\right)$ is invariantly universal? Similarly, is there a natural morphism relation $R$ which is complete analytic (or even invariantly universal when paired with some natural equivalence relation $\left.E \subseteq E_{R}\right)$ but such that $\left(R, E_{R}\right)$ is not invariantly universal? In particular, if $S$ is the embeddability relation between countable graphs or one of the morphism relations considered in Section 5 is it true that $\left(S, E_{S}\right)$ is invariantly universal?

Notice that Theorem 4.2 cannot be used to answer Question 6.4 If $S$ is complete for the class of analytic quasi-orders, then $E_{S}$ is complete for the class of analytic equivalence relations, and hence it is not Borel reducible to an orbit equivalence relation. 


\section{REFERENCES}

[BK96] Howard Becker and Alexander S. Kechris. The descriptive set theory of Polish group actions, volume 232 of London Mathematical Society Lecture Note Series. Cambridge University Press, Cambridge, 1996. MR 1425877 (98d:54068)

[Cam05a] Riccardo Camerlo. Universal analytic preorders arising from surjective functions. Fund. Math., 187(3):193-212, 2005. MR2213934 (2007f:03066)

[Cam05b] Riccardo Camerlo. Universality of embeddability relations for coloured total orders. Order, 22(3):289-300 (2006), 2005. MR2212690 (2007e:06001)

[CDM05] Riccardo Camerlo, Udayan B. Darji, and Alberto Marcone. Classification problems in continuum theory. Trans. Amer. Math. Soc., 357(11):4301-4328 (electronic), 2005. MR 2156712(2006f:03077)

[FMR11] Sy-David Friedman and Luca Motto Ros. Analytic equivalence relations and biembeddability. J. Symbolic Logic, 76(1):243-266, 2011. MR2791347 (2012b:03128)

[FS89] Harvey Friedman and Lee Stanley. A Borel reducibility theory for classes of countable structures. J. Symbolic Logic, 54(3):894-914, 1989. MR.1011177 (91f:03062)

[GK03] Su Gao and Alexander S. Kechris. On the classification of Polish metric spaces up to isometry. Mem. Amer. Math. Soc., 161(766):viii+78, 2003. MR.1950332(2004b:03067)

[Kau00] Robert Kaufman. PCA sets and convexity. Fund. Math., 163(3):267-275, 2000. MR.1758328 (2001d:54031)

[Kec95] Alexander S. Kechris. Classical descriptive set theory, volume 156 of Graduate Texts in Mathematics. Springer-Verlag, New York, 1995. MR1321597 (96e:03057)

[LR05] Alain Louveau and Christian Rosendal. Complete analytic equivalence relations. Trans. Amer. Math. Soc., 357(12):4839-4866 (electronic), 2005. MR2165390 (2006d:03081)

[MR04] Alberto Marcone and Christian Rosendal. The complexity of continuous embeddability between dendrites. J. Symbolic Logic, 69(3):663-673, 2004. MR2078915 (2005e:03106)

[MR11] Luca Motto Ros. On the complexity of the relations of isomorphism and biembeddability. Proc. Amer. Math. Soc., 140 (2012), no. 1, 309-323. MR2833542 (2012f:03095)

Dipartimento di Matematica, Politecnico di Torino, corso Duca degli Abruzzi 24, 10129 TORINO, ITALY

E-mail address: camerlo@calvino.polito.it

Dipartimento di Matematica e Informatica, Università di Udine, viale delle Scienze 206, 33100 Udine, ITALY

E-mail address: alberto.marcone@dimi.uniud.it

Kurt Gödel Research Center for Mathematical Logic, University of Vienna, Währinger Strasse 25, A-1090 Vienna, Austria

Current address: Albert-Ludwigs-Universität Freiburg, Mathematisches Institut - Abteilung für Mathematische Logik, Eckerstraße, 1, D-79104 Freiburg im Breisgau, Germany

E-mail address: luca.motto.ros@math.uni-freiburg.de 